\title{
GEOMETRIC GROUPS AND WHITEHEAD TORSION
}

\author{
BY \\ E. H. CONNELL AND JOHN HOLLINGSWORTH( $\left.{ }^{1}\right)$
}

The purpose of this paper is to define "geometric groups" and to relate them to various problems in topology. This relation is exhibited through Conjectures I and II. It will be shown that Conjecture I implies Conjecture II and that Conjecture II implies the topological invariance of Whitehead torsion. Conjecture II is true for 2-complexes, and this implies that if $K$ and $N$ are finite connected complexes, $L \subset K$ is a subcomplex with $\operatorname{dim} L \leqq 2$, and $f: K \rightarrow N$ is a homeomorphism with $f \mid K-L$ p.w.l., then $f$ is a simple homotopy equivalence. Another corollary is that if $K$ is a 2-complex contained in a p.w.l. manifold $M^{n}, U^{n}$ is a compact p.w.l. submanifold, $K \subset U \subset M$, and $U \varepsilon$-deforms to $K$, then $K \subset U$ is a simple homotopy equivalence and, thus, if $n \geqq 6, U$ is a regular neighborhood of $K$. Finally, for any finitely presented group $\pi$ with Wh $(\pi) \neq 0, \exists$ an $h$-cobordism $W$ with $\pi_{1}(W)=\pi$ which is not topologically trivial.

Geometric groups are related to other problems in topology and some of these are mentioned without proof in the appendix. For example, Conjecture I implies that compact ANRs of finite dimension have the homotopy type of finite complexes. Conjecture II has a noncompact analogue and since the difficulties are local, there is essentially nothing new here (Conjecture II is true for infinite 2-complexes). This noncompact form of Conjecture II implies the following: If $f: R^{n} \rightarrow R^{n}$ is a homeomorphism $(n \geqq 5)$ such that $f \times \mathrm{Id}: R^{n} \times R^{k} \rightarrow R^{n} \times R^{k}$ is stable, then $f$ is stable. The final note of the appendix implies the following: Suppose $\left(a_{i, j}\right)$ is an infinite matrix with integer entries, and that it and its inverse are band matrices, i.e., bounded about the diagonal. Then $\left(a_{i, j}\right)$ can be diagonalized by row operations. This is a nongeometric analogue of Conjecture II for the infinite complex $R^{1}$.

Notation, definitions, and conjectures. Although the results of this paper hold for CW complexes, their use has been avoided whenever possible to simplify the proofs. Unless otherwise stated, the word "complex" means "simplicial complex". If $K$ is a complex, $K \subset R^{n}$ means that $K$ is topologically embedded in $R^{n}$. This is important because in Lemma $5, K$ and $N$ are homeomorphic complexes embedded in essentially the same manner. If $K$ and $N$ are combinatorially distinct, they cannot each be simplicially embedded. In general, $K$ will be embedded in $R^{n}$ simply because $R^{n}$ is a pleasant metric space in which geometric construction may be performed.

Received by the editors March 16, 1966.

( ${ }^{1}$ ) This research was supported by the Sloan Foundation and NSF Grant GP-4055. 
Suppose $X$ is any metric space. If $A \subset B \subset X$ then $d(A)$ will be the diameter of $A$. If $x, y \in X$, then $d(x, y)$ is the diameter of the set $\{x, y\}$, i.e., the distance from $x$ to $y$. If $\delta>0, V(A, B, \delta)$ is defined to be $\{x \in B: d(A, x) \leqq \delta\}$. If $B=X$ and the meaning is clear, this may be written $V(A, \delta)$. The interior of $A$, where $A$ is considered as a subspace of $B$, is written Int $(A, B)$. The complement of $A$ in $B$ is written $\mathrm{Cm}(A, B)$ and the closure of $A$ in $B$ is written $\mathrm{Cl}(A, B)$. If the set $B$ is clear from context, these will be written $\operatorname{Int}(A), \mathrm{Cm}(A)$, and $\mathrm{Cl}(A)$ resp. The statement that $A$ is a $\delta$-deformation retract of $B$ means $\exists$ a map $h: B \times I \rightarrow B$ such that $h(x, 0)=x, h(x, 1) \in A$, and $d[h(x, I)]<\delta$ for all $x \in B$ and $h(x, t)=x$ for all $x \in A, t \in I$.

Definition of Geometric Group. Suppose $X$ is any metric space. The statement that $G$ is a geometric group associated with $X$ means that $\exists$ a finite collection $\left\{p_{i}\right\}_{i=1}^{v}$ where each $p_{i} \in X$ and that $G$ is the free group generated by the symbols $p_{i}$ (free group always means free abelian group). Even if the point $p_{i}$ is the same as the point $p_{j}$, they nevertheless represent distinct generators of $G$ because, when $i \neq j$, they are different symbols. This is a technicality-the reader should think of the points as being distinct. If $r$ is an integer, $1 \leqq r \leqq v$, and $g_{r} \in G$, then $g_{i, r}, 1 \leqq i \leqq v$, is the unique integer defined by the equation $g_{r}=g_{1, r} p_{1}+g_{2, r} p_{2}+\cdots+g_{v, r} p_{v}$. If $S \subset X$, then $G(S)=$ the subgroup of $G$ generated by $\left\{p_{i}: p_{i} \in S\right\}$. The symbol " $p_{i}$ " is used in two distinct ways and the meaning will be clear from context. For example, $p_{i} \in G(S)$ means $p_{i}=1 p_{i}$ is an element of the group $G ; p_{i} \in S$ means $p_{i}$ is a point of $X$. Note that $G=G(X)$ and if $S \subset X, G=G(S) \oplus G(\mathrm{Cm}(S))$. If none of the points $p_{i}$ are in $S, G(S)=0 \in G$. If $S_{1}, S_{2} \subset X$ then $G\left(S_{1}\right) \cap G\left(S_{2}\right)=G\left(S_{1} \cap S_{2}\right)$ and $G\left(S_{1}\right)+G\left(S_{2}\right)=G\left(S_{1} \cup S_{2}\right)$. If $A$ is a subset of $G$, the carrier of $A$ is the subset of $X$ defined by $\mathrm{Ca}(A)=\left\{p_{j}: \exists a \in A\right.$ such that when $a$ is expressed as a linear combination of the $p_{i}$, the coefficient of $p_{j}$ is nonzero\}. Thus $A \subset G(\mathrm{Ca}(A))$.

Definition of $\delta$-Basis. Suppose $\left\{a_{j}\right\}_{j=1}^{v}$ is a basis for $G$ (basis means free abelian basis). If $\delta>0$, the statement that $\left\{a_{j}\right\}$ is a $\delta$-basis means

(1) $d\left[\mathrm{Ca}\left(a_{j}\right)\right]<\delta$ for $j=1,2, \ldots, v$.

(2) Any $p_{i} \in G$ is a linear combination of $\left\{a_{j}: \mathrm{Ca}\left(a_{j}\right) \subset V\left(p_{i}, X, \delta\right)\right\}$.

The elements $a_{j}$ determine a set of integers $a_{i, j}$ by the equation $a_{j}=a_{1, j} p_{1}+\cdots$ $+a_{v, j} p_{v}$. Thus a basis determines a nonsingular integral matrix $\left(a_{i, j}\right)$. Conversely, given a nonsingular matrix $\left(a_{i, j}\right)$ of rank $v$, there is determined a basis $\left\{a_{j}\right\}$ by the same formula above. The matrix $\left(a_{i, j}\right)$ determines an isomorphism of $G$ onto itself. This automorphism is also written as $\left(a_{i, j}\right)$ and is determined by the formula $\left(a_{i, j}\right) p_{t}=a_{t}$.

Suppose $T$ is an automorphism on $G$. The statement that $T$ is $\delta$-blocked means that $\exists$ a finite collection $S_{1}, S_{2}, \ldots, S_{r}$, of disjoint subsets of $X$ with $G=$ $G\left(S_{1} \cup S_{2} \cup \ldots \cup S_{r}\right), d\left(S_{i}\right)<\delta$, and $T\left(G\left(S_{i}\right)\right)=G\left(S_{i}\right)$ for $i=1,2, \ldots, r$. A matrix is said to be $\delta$-blocked if the automorphism it determines is $\delta$-blocked. It should be noted that the transformation determined by a matrix depends upon the ordering of the set $\left\{p_{i}\right\}$, i.e., if this set is reindexed, the same matrix will determine a differ- 
ent transformation on $G$ which may no longer be $\delta$-blocked. Therefore when a matrix is said to be $\delta$-blocked, it means with respect to some definite ordered basis $\left\{p_{i}\right\}$. It is obvious that if $T$ is $\delta$-blocked, then $\left\{T\left(p_{i}\right)\right\}$ forms a $\delta$-basis. Thus the concept of " $\delta$-blocked" is a strong special form of the concept of " $\delta$-basis". The following proposition is appropriate at this stage.

Proposition 0. Suppose $G$ is a geometric group associated with $X$ and generated by $\left\{p_{i}\right\}_{i=1}^{v}$.

(a) If $T$ is an automorphism on $G$ with $\mathrm{Ca}\left[T\left(p_{i}\right)\right] \subset V\left(p_{i}, X, \delta\right)$ and $\mathrm{Ca}\left[T^{-1}\left(p_{i}\right)\right]$

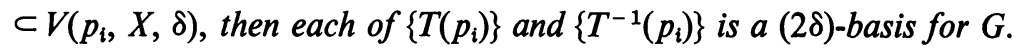

(b) If $\left\{a_{i}\right\}$ is a $\delta$-basis for $G$, then $\exists a \delta$-basis $\left\{b_{i}\right\}$ obtained by relabeling the $a_{i}$, such that the automorphism $T$ defined by $T\left(p_{i}\right)=b_{i}$ has the properties $\mathrm{Ca}\left[T\left(p_{i}\right)\right]$ $\subset V\left(p_{i}, X, \delta\right), \mathrm{Ca}\left[T^{-1}\left(p_{i}\right)\right] \subset V\left(p_{i}, X, \delta\right)$, and $\left\{T^{-1}\left(p_{i}\right)\right\}$ is a $(2 \delta)$-basis for $G$.

Proof of (a), Suppose $\mathrm{Ca}\left[T\left(p_{i}\right)\right]$ and $\mathrm{Ca}\left[T^{-1}\left(p_{i}\right)\right] \subset V\left(p_{i}, \delta\right)$. Then $d\left[\mathrm{Ca} T\left(p_{i}\right)\right]$ $<2 \delta$. Write $p_{i}=d_{1} T\left(p_{1}\right)+d_{2} T\left(p_{2}\right)+\cdots+d_{v} T\left(p_{v}\right)$, and thus $T^{-1}\left(p_{i}\right)=d_{1} p_{1}+d_{2} p_{2}+$ $\cdots+d_{v} p_{v}$. Since $\mathrm{Ca}\left[T^{-1}\left(p_{i}\right)\right] \subset V\left(p_{i}, \delta\right), d_{j} \neq 0 \Rightarrow p_{j} \in V\left(p_{i}, \delta\right) \Rightarrow T\left(p_{j}\right) \subset V\left(p_{i}, 2 \delta\right)$. Therefore $p_{i}$ is a linear combination of $\left\{T\left(p_{j}\right): \mathrm{Ca}\left[T\left(p_{j}\right)\right] \subset V\left(p_{i}, 2 \delta\right)\right\}$ and this

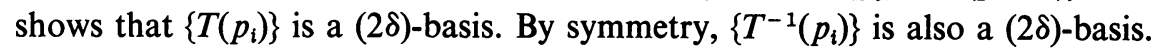

Proof of (b). Suppose $\left\{a_{i}\right\}$ is a $\delta$-basis and, as always, let $\left(a_{i, j}\right)$ be the matrix determined by the automorphism $\left(a_{i, j}\right) p_{t}=a_{t}$. Since $\left(a_{i, j}\right)$ is nonsingular, $\exists$ a matrix $\left(b_{i, j}\right)$ obtained from $\left(a_{i, j}\right)$ by interchanging columns, such that the diagonal entries $b_{i, i} \neq 0$ for $i=1,2, \ldots, v$ (this elementary fact is assumed). As always, let $b_{t} \in G$ be defined by $b_{t}=\left(b_{i, j}\right) p_{t}=T\left(p_{t}\right)$. (The set $\left\{b_{t}\right\}$ is simply a relabeling of the set $\left\{a_{t}\right\}$.) Since $p_{t} \in \mathrm{Ca}\left(b_{t}\right)$ and $d\left[\mathrm{Ca}\left(b_{t}\right)\right]<\delta$, it follows that $\mathrm{Ca}\left[T\left(p_{t}\right)\right]=\mathrm{Ca}\left(b_{t}\right) \subset V\left(p_{t}, \delta\right)$.

Since $\left\{b_{t}\right\}$ is a $\delta$-basis, $p_{t}=d_{1} b_{1}+d_{2} b_{2}+\cdots+d_{v} b_{v}$ where $d_{i} \neq 0 \Rightarrow \mathrm{Ca}\left(b_{i}\right) \subset$ $V\left(p_{t}, \delta\right) . \quad T^{-1}\left(p_{t}\right)=d_{1} p_{1}+\cdots+d_{v} p_{v} \quad$ where $\quad d_{i} \neq 0 \Rightarrow p_{i} \in \mathrm{Ca}\left(b_{i}\right) \subset V\left(p_{t}, \delta\right)$. This

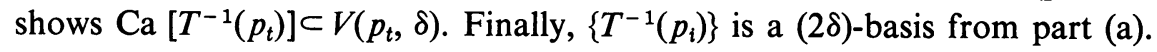

DEFINITION OF $\delta$-MAP. If $G=G(X)$ is a geometric group and $H$ is a free abelian group, a $\delta$-map $f: G \rightarrow H$ is a homomorphism onto satisfying (1) and (2) below (properties (3) and (4) follow from (2)).

(1) If $S \subset X, f[G(S)] \cap f[G(\mathrm{Cm} V[S, \delta])]=0$.

(2) If $S_{1}$ and $S_{2}$ are sets with $X=S_{1} \cup S_{2}$ and $S_{1} \cap S_{2}=\varnothing$, then for any $g \in G\left(S_{1}\right)$ and any nonzero integer $n$ with $f(n g) \in f\left[G\left(S_{2}\right)\right], \exists \bar{g} \in G\left[V\left(S_{1}, \delta\right) \cap V\left(S_{2}, \delta\right)\right]$ with $f(g)=f(\bar{g})$.

(3) If $S_{1}$ and $S_{2}$ are two sets with $X=S_{1} \cup S_{2}$ and $S_{1} \cap S_{2}=\varnothing$, then

$$
f\left[G\left(S_{1}\right)\right] \cap f\left[G\left(S_{2}\right)\right] \subset f\left(G\left[V\left(S_{1}, \delta\right) \cap V\left(S_{2}, \delta\right)\right]\right) .
$$

(4) If $S \subset X$ then $\exists$ a subgroup $G^{\prime} \subset G$ such that $G(S) \subset G^{\prime} \subset G[V(S, \delta)]$ and $H / f\left(G^{\prime}\right)$ has no torsion.

ObSERvation. Condition (2) implies conditions (3) and (4).

Proof. Suppose (2) is true. Condition (3) follows by setting $n=1$. To show (4) is true, let $S_{2}=S \subset X, S_{1}=\mathrm{Cm}\left(S_{2}, X\right), \pi: H \rightarrow H / f\left[G\left(S_{2}\right)\right]$ be the projection, and $T$ 
be the torsion subgroup of $H / f\left[G\left(S_{2}\right)\right]$. Let $A \subset G$ be a finite set such that $\pi f(A)=T$. Since $H=f\left[G\left(S_{1}\right)\right]+f\left[G\left(S_{2}\right)\right]$ and $\pi f\left[G\left(S_{2}\right)\right]=0, A$ may be chosen so that $A \subset G\left(S_{1}\right)$. Now by (2), $\exists B \subset G\left[V\left(S_{1}, \delta\right) \cap V\left(S_{2}, \delta\right)\right]$ with $f(B)=f(A)$. The proof is completed by setting $G^{\prime}=$ the subgroup of $G$ generated by $G\left(S_{2}\right) \cup B$. Then $G\left(S_{2}\right) \subset G^{\prime} \subset$ $G\left[V\left(S_{2}, \delta\right)\right], \pi f\left(G^{\prime}\right)=T$ and $H / f\left(G^{\prime}\right)$ has no torsion.

The connection between $\delta$-bases and $\delta$-maps is as follows: If $G=G(X)$ is a geometric group, $\left\{a_{i}\right\}$ is a $\delta$-basis of $G, A$ is a subgroup of $G$ generated by some subset of $\left\{a_{i}\right\}$, and $S \subset X$ with $A \subset G(S)$, then the map $\pi: G(S) \rightarrow G(S) / A$ is a

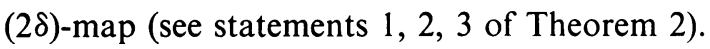

CONJECTURE $\mathrm{I}_{n}$. If $K$ is a finite complex, $\operatorname{dim} K \leqq n$, and $\varepsilon>0, \exists \delta>0$ such that: If $G$ is any geometric group associated with $K, H$ is a free abelian group, and $f: G \rightarrow H$ is a $\delta$-map, then $\exists\left\{g_{i}\right\}_{i=1}^{r} \subset G$ such that

(1) $\left\{f\left(g_{i}\right)\right\}_{i=1}^{r}$ is a free abelian basis for $H$,

(2) $d\left[\mathrm{Ca}\left(g_{i}\right)\right]<\varepsilon$ for $i=1,2, \ldots, r$,

(3) If $S \subset K$, then $f[G(S)] \subset$ the subgroup of $H$ generated by $\left\{f\left(g_{i}\right): \mathrm{Ca}\left(g_{i}\right)\right.$ $\subset V(S, K, \varepsilon)\}$.

Conjecture II $_{n}$. If $K$ is a finite complex, $\operatorname{dim} K \leqq n$, and $\varepsilon>0, \exists \delta>0$ such that: If $G$ is any geometric group associated with $K$, generated by $\left\{p_{i}\right\}_{i=1}^{v}$, and $\left\{a_{i}\right\}_{i=1}^{v}$ is any $\delta$-basis for $G$, then $\exists$ a sequence $\left(\gamma^{0}\right),\left(\gamma^{1}\right), \ldots,\left(\gamma^{n+1}\right)$ of $v \times v$ matrices satisfying

(i) Each $\left(\gamma^{i}\right)$ is $\varepsilon$-blocked for $i=1,2, \ldots, n+1$, and $\left(\gamma^{0}\right)$ is obtained from the identity matrix by interchanging columns.

(ii) $\left(\gamma^{n+1}\right)\left(\gamma^{n}\right) \cdots\left(\gamma^{1}\right)=\left(a_{i, j}\right)\left(\gamma^{0}\right)$ where $\left(a_{i, j}\right)$ is the matrix associated with the basis $\left\{a_{i}\right\}$.

The connection between Conj. I and Conj. II is as follows: If $G=G(K)$ is a geometric group, $\left\{a_{i}\right\}$ is a $\delta$-basis of $G, A$ is a subgroup of $G$ generated by some subset of $\left\{a_{i}\right\}$, and $S \subset K$ with $A \subset G(S)$, then as mentioned previously, the map

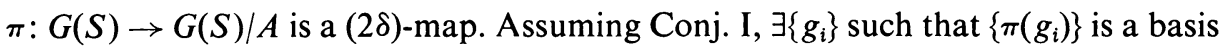
for $G(S) / A$. Then $\left\{\pi\left(g_{i}\right)\right\}$ together with the basis for $A$ forms a $\left(2 \delta+\delta_{1}\right)$-basis for $G(S)$. Thus it is possible to "block off" the basis for $G$ and it is this process which allows the proof that Conj. $\mathrm{I}_{n} \Rightarrow$ Conj. $\mathrm{II}_{n+1}$. Each of these conjectures becomes nontrivial in a hurry-when $n=1$ for example. However the following proposition is trivial.

Proposition 1. Conjectures $\mathrm{I}_{0}$ and $\mathrm{II}_{0}$ are true.

Proof of Conj. $\mathrm{I}_{0}$. Suppose $K$ is a 0 -complex, $K=\left\{x_{1}, x_{2}, \ldots, x_{n}\right\}$, and $\varepsilon>0$. Let $\delta>0$ such that $d\left(x_{i}, x_{j}\right)>\delta$ whenever $i \neq j$. Let $G=G(K)$ be generated by $\left\{p_{i}\right\}_{i=1}^{v}$ and $f: G \rightarrow H$ a $\delta$-map. Let $G=G_{1} \oplus G_{2} \oplus \cdots \oplus G_{n}$ where $G_{i}$ is the subgroup of $G$ generated by $\left\{p_{j}: p_{j}=x_{i}\right\}$ (remember that the $p_{i}$ may not be distinct). Since $f$ is a $\delta$-map, $H=f\left(G_{1}\right) \oplus \cdots \oplus f\left(G_{n}\right)$. Let $C_{i}$ be a finite subset of $G_{i}$ such that $f\left(C_{i}\right)$ is a basis for $f\left(G_{i}\right)$. Then $\bigcup_{i \leqq i \leqq n} C_{i}$ is the desired subset of $G$. The proof of Conj. $\mathrm{II}_{0}$ is equally trivial and is omitted.

Conjecture II implies the topological invariance of Whitehead torsion. The 
purpose of this section is to prove Lemma 5 below. The five lemmas presented are obvious and the proofs are elementary (but tedious) exercises in general topology. The first lemma shows how a $\delta$-basis arises from a geometric situation.

LEMMA 1. Hypothesis. $K \subset R^{2 k+5}$ is a connected $k$-complex and $Y \subset K$ is a connected subcomplex. $M \subset R^{2 k+5}$ is a $\mathrm{CW}$ complex containing $Y$ as a subcomplex and $M \cap K=Y$. $(M, Y)$ contains cells only in dimensions $k+1$ and $k+2:$ the $(k+1)$-cells are $(k+1)$-spheres $\left\{S_{1}, S_{2}, \ldots, S_{v}\right\}$ attached to $Y$ at points $\left\{p_{1}, p_{2}, \ldots\right.$, $\left.p_{v}\right\}$; the $(k+2)$-cells $\left\{D_{1}, D_{2}, \ldots, D_{v}\right\}$ are attached by maps $f_{i}: \partial D_{i} \rightarrow\left(Y \cup S_{1}\right.$ $\left.\cup S_{2} \cup \cdots \cup S_{v}\right) . Y$ is a $\delta$-deformation retract of $M, d\left(S_{i}\right)<\delta$, and $d\left(D_{i}\right)<\delta$ for $i=1,2, \ldots, v . G$ is the geometric group associated with $Y$ that is generated by the symbols $\left\{p_{i}\right\}_{i=1}^{v}$. G also has a basis $\left\{a_{i}\right\}_{i=1}^{v}$ determined as follows: Let $\gamma_{i}$ be a generator of $H_{k+1}\left(S_{i} \cup Y, Y\right) \approx Z$ and $\Delta_{j}$ be a generator of $H_{k+2}\left(D_{j}, \partial D_{j}\right)$. Then the image of $\Delta_{j}$ under the map

$$
\left(f_{j}\right)_{*} \partial: H_{k+2}\left(D_{j}, \partial D_{j}\right) \rightarrow H_{k+1}\left(\partial D_{j}\right) \rightarrow H_{k+1}\left(Y \cup S_{1} \cup S_{2} \cup \cdots \cup S_{v}, Y\right)
$$

is $a_{1, j} \gamma_{1}+a_{2, j} \gamma_{2}+\cdots+a_{v, j} \gamma_{v}$. Then $a_{j} \in G$ is defined by $a_{j}=a_{1, j} p_{1}+a_{2, j} p_{2}+\cdots$ $+a_{v, j} p_{v}$.

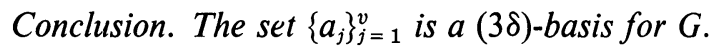

Proof. Since $d\left(D_{j}\right)<\delta<3 \delta$, it follows immediately that $d\left[\mathrm{Ca}\left(a_{j}\right)\right]<3 \delta$. Since $S_{i}$ deforms to $Y$ in $V\left(p_{i}, M, 2 \delta\right)$, it follows that $S_{i}$ deforms to $Y$ in

$$
Y \cup\left(S_{1} \cup S_{2} \cup \cdots \cup S_{v}\right) \cup\left[D_{j}: D_{j} \subset V\left(p_{i}, M, 3 \delta\right)\right\} .
$$

Therefore $p_{i} \in G$ is generated by a combination of $\left\{a_{j}: \mathrm{Ca}\left(a_{j}\right) \subset V\left(p_{i}, Y, 3 \delta\right)\right\}$. This completes the proof.

Suppose $f: K \rightarrow N$ is a homeomorphism and $M_{f}$ is its mapping cylinder. Then $M_{f}$ is homeomorphic to $K \times I, K$ is a deformation retract of $M_{f}$, and this deformation, when projected onto $N$, is the identity. If $h$ is a map approximating $f$, then $K$ is a deformation retract of $M_{h}$, and this deformation, when projected onto $N$, approximates the identity. The next lemma is a relative version of that fact.

Lemma 2. Let $N \subset R^{n}$ be a finite complex. If $\varepsilon>0, \exists \delta>0$ such that whenever

(i) $K$ is any finite complex and $W$ is any finite subcomplex of some subdivision of $K$,

(ii) $f: K \rightarrow N$ is a homeomorphism which is p.w.l. on $W$, and

(iii) $h: K \rightarrow N$ is a p.w.l. map with $h|W=f| W$ and

$$
d(h(x), f(x))<\delta \quad \text { for } x \in K,
$$

then $\exists$ a map $H: M_{h} \times I \rightarrow M_{h}$ such that

(a) $H(z, 0)=z$ and $H(z, 1) \in K$ for all $z \in M_{h}$ and $H(x, u)=x$ for all $x \in K \subset M_{h}$ and $u \in[0,1]$,

(b) $d\left[\pi_{N}(H[z, I])\right]<\varepsilon$ for all $z \in M_{h}$ and $\pi_{N}[(x, t), u]=f(x)=h(x)$ for $x \in W$ and $t, u \in[0,1]$. 
(The mapping cylinder over $W$ is just $W \times I$. Thus $(x, t)$ above is in $M_{h}$.)

Proof. Suppose $N \subset R^{n}$ and $\varepsilon>0$. Let $0 \subset R^{n}$ be an open set, $N \subset 0$, and $r: 0 \rightarrow N$ a retraction: Let $\delta>0$ such that if $x, y \in N$ and $d(x, y)<\delta$, and $T$ is the straight line interval joining $x$ to $y$, then $T \subset 0$ and $d[r(T)]<\varepsilon / 3$. This number $\delta$ will satisfy the conclusion of the lemma. Let $(K, W), f$, and $h$ be given satisfying (i), (ii), and (iii). Since $d(f, h)<\delta, f$ and $h$ are $\varepsilon / 3$-homotopic relative to $W$, i.e., $\exists g: K \times I \rightarrow N \times I$ with

$$
\begin{aligned}
& g(x, 0)=(f(x), 0), \\
& g(x, 1)=(h(x), 1), \\
& d\left[\pi_{N}(g[x, I])\right]<\varepsilon / 3 \text { and } \\
& \pi_{1}(g(x, t))=t \text { for } x \in K, t \in[0,1], \text { and also } \\
& g(x, t)=(f(x), t)=(h(x), t) \text { for } x \in W, t \in[0,1] .
\end{aligned}
$$

Construct the mapping cylinder $M_{g}=\left[(K \times I) \times I \cup_{g} N \times I\right]$ and identify $M_{f}$ with $(K \times 0) \times I \cup N \times 0$, and $M_{h}$ with $(K \times 1) \times I \cup N \times 1$. Now $(I \times 0) \cup(0 \times I) \cup(I \times 1)$ is a deformation retract of $I \times I$. Any such deformation induces a map $p_{1}: M_{g} \times I$ $\rightarrow M_{g}$ which deforms $M_{g}$ into

$$
\begin{aligned}
{[(K \times I \times 0) \cup N \times I] \cup } & {[(K \times 0 \times I) \cup N \times I] \cup[(K \times I \times 1) \cup N \times I] } \\
& =(K \times I \times 0) \cup M_{f} \cup N \times I=X
\end{aligned}
$$

with $d\left[\pi_{N}\left(p_{1}(z, I)\right)\right]<\varepsilon / 3$ for all $z \in M_{g}$. Here $\pi_{N}$ represents the natural projection of $M_{g}$ to $N \times I$ followed by its projection onto $N$.

Now $N \times I$ deforms to $N \times 0 \subset M_{f}, M_{f}$ deforms to $(K \times 0 \times 0) \subset(K \times I \times 0)$ because $f$ is a homeomorphism, and $K \times I \times 0$ deforms to $K \times 1 \times 0$ which is the base of $M_{h}$. These three deformations performed in sequence yield $p_{2}: X \times I \rightarrow X$, a deformation of $X$ onto $K \times I \times 0 \subset M_{h}$ with $\pi_{N}(z \times I)=\pi_{N}(z)$ for any $z \in X$.

Define $p: M_{h} \times I \rightarrow M_{g}$ by

$$
\begin{aligned}
p(z, t) & =p_{1}(a, 2 t) & & \text { for } 0 \leqq t \leqq \frac{1}{2}, \\
& =p_{2}\left(p_{1}(z, 1), 2 t-1\right) & & \text { for } \frac{1}{2} \leqq t \leqq 1,
\end{aligned}
$$

and note that

and

$$
\begin{gathered}
p(z, 0)=z, \quad p(z, 1) \in K \times 1 \times 0 \subset M_{h} \subset M_{g}, \\
d\left(\pi_{N}[p(z, I)]\right)<\varepsilon / 3 \quad \text { all } z \in M_{h}
\end{gathered}
$$

$$
\pi_{N}(x, t, u)=x \quad \text { all }(x, t, u) \in W \times I \times I .
$$

A retraction $r: M_{g} \rightarrow M_{h}$ may be defined as follows: For $(x, t, u) \in K \times I \times I$

$$
\begin{aligned}
r(x, t, u) & =(x, 1,(1-t)+u) & & \text { when }(1-t)+u \leqq 1, \\
& =(x, 1,1) & & \text { when }(1-t)+u \geqq 1 .
\end{aligned}
$$

For $(y, t) \in N \times I, r(y, t)=(y, 1)$. Note that $(x, 1,1)$ is identified with $(f(x), 1)$ $\in N \times I$ in $M_{h} \subset M_{g}$. Then $r$ satisfies $d\left(\pi_{N}(z), \pi_{N}[r(z)]\right)<\varepsilon / 3$ for $z \in M_{g}$. Also 
$\pi_{N}[r(z, t, u)]=\pi_{N}(x, t, u)=h(x)=f(x)$ for $(x, t, u) \in W \times I \times I$. The deformation $H: M_{h} \times I \rightarrow M_{h}$ desired in the conclusion of the lemma is given by $H=r p$.

Suppose $K$ is a deformation retract of $Q$. It is a classical theorem of J. H. C. Whitehead that $\exists M \supset K$ such that $(M, K)$ has cells in only two distinct dimensions, $M$ deforms to $K$, and the Whitehead torsion $\tau(M, K)=\tau(Q, K)$. The next lemma states that if $Q \delta$-deforms to $K$ then $M$ may be chosen to $\varepsilon$-deform to $K$. This is proved by working through Whitehead's proof and keeping track of distances. Since the proof has no instructional value whatsoever, it is omitted.

LEMma 3. Suppose $K \subset R^{2 k+5}$ is a finite connected $k$-complex, $Y \subset K$ is a connected subcomplex, and $\varepsilon>0$. Then $\exists \delta>0$ such that if $Q \subset R^{2 k+5}$ is any $(k+1)$-complex with $Q \cap K=Y$ which $\delta$-deforms to $Y$, then $\exists$ a CW complex $M \subset R^{2 k+5}$ containing $Y$ as a subcomplex, such that $M \cap K=Y$ and

(a) $M \varepsilon$-deforms to $Y$.

(b) $(M, Y)$ has cells only in dimensions $k+1$ and $k+2$ and the $(k+1)$-cells are attached trivially.

(c) If $\sigma$ is a cell of $(M, Y)$ then $d(\varepsilon)<\varepsilon$.

(d) The Whitehead torsion $\tau(M \cup K, K)=\tau(Q \cup K, K)$.

Suppose $K$ is a finite connected complex and $L \subset K$ is a subcomplex. Let $\delta_{1}>0$ such that any set of diameter $<\delta_{1}$ is contained in the star of some vertex. Suppose $G$ is a geometric group associated with $L$, generated by $\left\{p_{i}\right\}_{i=1}^{v}$, and $\left\{a_{i}\right\}_{i=1}^{v}$ is a $\delta$-basis for $G$ where $0<\delta<\delta_{1}$. Then the basis $\left\{a_{i}\right\}$ determines an element of the Whitehead group Wh $\left(\pi_{1}(K)\right)$ as follows: Join each $p_{i}$ to the base point by a path $P_{i}$. Join each $\mathrm{Ca}\left(a_{j}\right)$ to the base point by a path $A_{j}$-this is possible because Ca $\left(a_{j}\right) \subset$ the star of some vertex. For each $p_{i} \in \mathrm{Ca}\left(a_{j}\right),\left[P_{i}^{-1} A_{j}\right]$ will represent the element of $\pi_{1}(K)$ determined by the loop $P_{i}^{-1} A_{j}$.

The matrix $\left(a_{i j}\left[P_{i}^{-1} A_{j}\right]\right)$ determines an element of Wh $\left(\pi_{1}(K)\right)$. This element is independent of the ordering of the set $\left\{p_{i}\right\}$, the ordering of the set $\left\{a_{i}\right\}$, the paths $P_{i}$ and the paths $A_{j}$. It will be shown that if $\operatorname{dim} L \leqq n$ and Conj. $I_{n}$ is true, then for $\delta$ small enough, this element of $\mathrm{Wh}\left(\pi_{1}(K)\right)$ is 0 .

Lemma 4. Suppose $K$ is a finite connected compler and $L$ is a subcompler with $\operatorname{dim} L \leqq n$. Then if Conjecture $\mathrm{II}_{n}$ is true, $\exists \delta>0$ such that if $G$ is any geometric group associated with $L$, and $\left\{a_{i}\right\}$ is any $\delta$-basis for $G$, then the matrix $\left(a_{i, j}\left[P_{i}^{-1} A_{j}\right]\right)$ represents the 0 element in Wh $\left(\pi_{1}(K)\right)$.

Proof. Suppose $K$ is given. The proof is by induction on $\operatorname{dim} L$. Suppose $\operatorname{dim} L=0$. Then let $\delta>0$ be such that $\delta$ is less than the minimum distance between vertices of $L$. Then for any geometric group $G$ and any $\delta$-basis $\left\{a_{i}\right\}$, each $\mathrm{Ca}\left(a_{j}\right)$ = some $p_{i}$. Then the path $A_{j}$ can be chosen equal to the path $P_{i}$ and therefore the matrix $\left(a_{i, j}\left[P_{i}^{-1} A_{j}\right]\right)$ will have only integer entries and can be diagonalized by row and column operations, and will represent the 0 element of Wh $\left(\pi_{1}(K)\right)$.

Now suppose by induction that the lemma is true when the dimension of the subcomplex is $\leqq n$. Suppose Conjecture $\mathrm{II}_{n+1}$ is true and $\operatorname{dim} L=n+1$. Let $\varepsilon>0$ be 
such that the conclusion of the lemma holds for the subcomplex $L^{n}$, the $n$-skeleton of $L$. This means that if $G^{\prime}$ is any geometric group associated with $L^{n}$, then any $\varepsilon$-basis for $G^{\prime}$ determines 0 in Wh $\left(\pi_{1}(K)\right)$.

Let $O_{1}$ and $O_{2}$ be open subsets of $L$ with $L^{n} \subset O_{1}$ and $\mathrm{Cl}\left(O_{1}\right) \subset O_{2}$, and $r: O_{2} \rightarrow L^{n}$ a retraction with $d(r(x), x)<\varepsilon / 8$ for all $x \in O_{2}$. Let $\varepsilon_{1}>0$ such that $\varepsilon_{1}<\varepsilon / 16(n+2)$, $V\left(L^{n}, L, 4(n+2) \varepsilon_{1}\right) \subset O_{1}$, and $V\left(O_{1}, L, 4(n+2) \varepsilon_{1}\right) \subset O_{2}$.

By assumption, Conjecture $\mathrm{II}_{n+1}$ is true. Thus $\exists \delta, 0<\delta<\varepsilon_{1}$, such that the conclusion of Conjecture II is true for the complex $L$ and the positive number $\varepsilon_{1}$. This is to be the " $\delta$ " in the conclusion of this lemma. Let $G$ be a geometric group associated with $L$, generated by $\left\{p_{i}\right\}_{i=1}^{v}$ and containing a $\delta$-basis $\left\{a_{i}\right\}_{i=1}^{v}$. It must be shown that the basis $\left\{a_{i}\right\}$ determines the 0 element of Wh $\left(\pi_{1}(K)\right)$.

The paths $P_{i}$ and $A_{j}$ are to be selected satisfying the following: If $\sigma$ is an $(n+1)-$ simplex of $L, p_{i} \in \operatorname{Int} \sigma$, and $\mathrm{Ca}\left(a_{j}\right) \subset \operatorname{Int} \sigma$, then $\left[P_{i}^{-1} A_{j}\right]$ is the unit of $\pi_{1}(K)$. This is done by joining $\sigma$ to the base point by a path and using this single path to determine all $P_{i}$ and $A_{j}$ above. If $p_{i} \in L^{n}$, then $P_{i}$ is chosen arbitrarily. If $\mathrm{Ca}\left(a_{j}\right)$ is not contained in the interior of some $(n+1)$-simplex of $L$, then $A_{j}$ is chosen arbitrarily.

Since Conjecture $\mathrm{II}_{n+1}$ is true and $\left\{a_{i}\right\}$ is a $\delta$-basis, $\left(a_{i, j}\right)=\left(\alpha^{n+2}\right)\left(\alpha^{n+1}\right) \cdots\left(\alpha^{1}\right)$ where each $\left(\alpha^{i}\right)$ is $\varepsilon_{1}$-blocked. (Conjecture II gives this after $\left(a_{i, j}\right)$ is altered by interchanging columns. Since interchanging columns does not effect the induced element in Whitehead torsion, we suppose that $\left(a_{i, j}\right)$ itself satisfies this equation.) Let $S_{1}, S_{2}, \ldots, S_{r}$ be disjoint subsets of $L$ such that $G=G\left(S_{1} \cup S_{2} \cup \cdots \cup S_{r}\right)$, $\left(\alpha^{1}\right)\left[G\left(S_{t}\right)\right]=G\left(S_{t}\right)$, and $d\left(S_{t}\right)<\varepsilon_{1}$ for $t=1,2, \ldots, r$. Define a new matrix $\left(\bar{\alpha}^{1}\right)$ by the transformation formula: $\left(\bar{\alpha}^{1}\right)\left|G\left(S_{t}\right)=\left(\alpha^{1}\right)\right| G\left(S_{t}\right)$ when $S_{t} \subset \mathrm{Cm}\left(0_{1}, L\right)$ and $\left(\bar{\alpha}^{1}\right) \mid G\left(S_{t}\right)$ $=$ the identity, otherwise. Define similarly $\left(\bar{\alpha}^{2}\right), \ldots,\left(\bar{\alpha}^{n+2}\right)$, and let $(\bar{\alpha})=\left(\bar{\alpha}^{n+2}\right)$ $\cdot\left(\bar{\alpha}^{n+1}\right) \cdots\left(\bar{\alpha}^{1}\right)$. Finally, let $\left(\beta_{i, j}\right)=\left(a_{i, j}\left[P_{i}^{-1} A_{j}\right]\right)(\bar{\alpha})^{-1}$ and $\left(b_{i, j}\right)=\left(a_{i, j}\right)(\bar{\alpha})^{-1}$.

Note that for any $(n+1)$-simplex $\sigma$ of $L,\left(a_{i, j}\right)\left[G\left(\sigma \cap \mathrm{Cm}\left(O_{1}\right)\right)\right] \subset G(\operatorname{Int} \sigma)$. This is true because $V\left(L^{n}, L, 4(n+2) \varepsilon_{1}\right) \subset O_{1}$ and $\left(a_{i, j}\right)$ is the product of $(n+2)$ matrices, each of which is $\varepsilon_{1}$-blocked. Therefore $p_{t} \in \mathrm{Cm}\left(O_{1}\right)$ implies $\left(a_{i, j}\left[P_{i}^{-1} A_{j}\right]\right) p_{t}=$ $\left(a_{i, j}\right) p_{t}=a_{t}$, i.e., no fundamental group elements are involved. Since $(\bar{\alpha})^{-1}$ is the identity on $G\left(O_{1}\right),(\bar{\alpha})^{-1}$ does not effect the matrix $\left(a_{i, j}\left[P_{i}^{-1} A_{j}\right]\right)$ whenever nontrivial elements of the fundamental group appear.

The basic properties of $\left(\beta_{i, j}\right)$ are:

(a) $\left(\beta_{i, j}\right)$ represents the same element of Wh $\left(\pi_{1}(K)\right)$ as does $\left(a_{i, j}\left[P_{i}^{-1} A_{j}\right]\right)$.

(b) $\left(\beta_{i, j}\right)$ arises from the basis $\left\{\left(b_{i, j}\right) p_{t}\right\}$.

Property (a) follows from the fact that $(\bar{\alpha})^{-1}$ has integer entries and is thus 0 in the Whitehead group. Property (b) is obvious from the remarks above. The basic properties of $\left(b_{i, j}\right)$ are:

(1) Whenever $p_{t} \in G\left[\mathrm{Cm}\left(O_{2}\right)\right], \quad\left(b_{i, j}\right) p_{t}=p_{t}$. (In this case $b_{i, t}=\beta_{i, t}=0$ when $i \neq t$ and $b_{t, t}=\beta_{t, t}=1$.)

(2) $\left(b_{i, j}\right) p_{t}$ and $\left(b_{i, j}\right)^{-1} p_{t} \subset G\left[V\left(p_{t}, L, 4(n+2) \varepsilon_{1}\right)\right] \subset G\left[V\left(p_{t}, L, \varepsilon / 4\right)\right]$.

(3) $\left(b_{i, j}\right)\left[G\left(O_{2}\right)\right]=G\left(O_{2}\right)$. 
Property (1) follows from $\left(a_{i, j}\right)^{-1}\left(p_{t}\right)=(\bar{\alpha})^{-1}\left(p_{t}\right)$ for each $p_{t} \in G\left[\mathrm{Cm}\left(O_{2}\right)\right]$. Property (2) follows from the fact that $\left(b_{i, j}\right)$ and $\left(b_{i, j}\right)^{-1}$ are the products of $2(n+2)$ matrices, each of which is $\varepsilon_{1}$-blocked, and the fact that $\varepsilon_{1}<\varepsilon / 16(n+2)$. Property (3) follows from (1).

If row $i$ and column $j$ of a matrix are each composed of zeros except for a "one" in the $(i, j)$ place, then this row and column may be removed from the matrix without changing its class in the Whitehead group. Thus if $\left(\beta_{i, j}^{\prime}\right)$ is the matrix determined by $\left(\beta_{i, j}\right)$ restricted to $G\left(O_{2}\right)$, then $\left(\beta_{i, j}^{\prime}\right)$ represents the same class in Wh $\left(\pi_{1}(K)\right)$ as does $\left(\beta_{i, j}\right)$. Let $\left(b_{i, j}^{\prime}\right)$ be the matrix determined by $\left(b_{i, j}\right)$ restricted to $G\left(O_{2}\right)$. Then $\left(b_{i, j}^{\prime}\right)$ still satisfies (2) and (3) above.

Let $G^{\prime}$ be the geometric group associated with $L^{n}$ and generated by the symbols $\left\{r\left(p_{i}\right): p_{i} \in G\left(O_{2}\right)\right\}$. Now $\left(b_{i, j}^{\prime}\right)$, considered as a transformation of $G^{\prime}$ onto itself, satisfies $\left(b_{i, j}^{\prime}\right)\left[r\left(p_{t}\right)\right]$ and $\left(b_{i, j}^{\prime}\right)^{-1}\left[r\left(p_{t}\right)\right] \subset V\left[r\left(p_{t}\right), L^{n}, \varepsilon / 2\right]$. This follows from (2) and the fact that $d(r(x), x)<\varepsilon / 8$ for $x \in O_{2}$. Therefore by Proposition 0 , part (a), $\left(b_{i, j}^{\prime}\right)$ arises from an $\varepsilon$-basis. By induction and the manner in which $\varepsilon$ was chosen, it follows that $\left(b_{i, j}^{\prime}\right)$ determines the 0 element in Wh $\left(\pi_{1}(K)\right)$. It follows from (b) above that $\left(b_{i, j}^{\prime}\right)$ determines the element $\left(\beta_{i, j}^{\prime}\right)$, and the proof is complete.

The purpose of the four preceding lemmas is to prove Lemma 5 below. Lemma 5 is a relative version of the classical conjecture that homeomorphisms induce simple homotopy equivalences. Here is the idea of the proof: Suppose $f: K \rightarrow N$ is a homeomorphism. Let $h: K \rightarrow N$ be a p.w.l. map which approximates $f$. Using a short mapping cylinder (instead of one of length "one") and Lemma 2, the mapping cylinder $M_{h}$ will deform to its base $K$ through a small deformation. By Lemma 3, it may be assumed that $\left(M_{h}, K\right)$ has cells in only two dimensions. Using Lemma 1 , this determines a geometric group associated with $K$ and a $\delta$-basis. It follows from Lemma 4 and the assumption that Conjecture II is true, that this basis induces the 0 element in Wh $\left(\pi_{1}(K)\right)$.

Lemma 5. Suppose Conjecture $\mathrm{II}_{n}$ is true, $K$ is a finite connected $k$-complex, and $L$ is a connected subcomplex of $K$ with $\operatorname{dim} L \leqq n$. Then there exists a subcomplex $X$ of some subdivision of $K, L \subset \operatorname{Int}(X, K)$, such that if $N$ is any finite complex and $f: K \rightarrow N$ is any homeomorphism with $f \mid \mathrm{Cm}(\operatorname{Int} X, K)$ p.w.l., then $f$ is a simple homotopy equivalence.

Proof. Suppose $K$ is topologically embedded in $R^{2 k+1}$. By Lemma 4, there exists $\varepsilon_{1}>0$ such that $G$ is any geometric group associated with $L$ and $\left\{a_{i}\right\}$ is any $\varepsilon_{1}$-basis for $G$, then the matrix $\left(a_{i, j}\left[P_{i}^{-1} A_{j}\right]\right)$ represents the 0 element in Wh $\left(\pi_{1}(K)\right)$. Let $K_{1}$ be a subdivision of $K, Y=\mathrm{St}\left(L_{1}, K_{1}\right)$, and $r: Y \rightarrow L$ be a retraction. Suppose the subdivision $K_{1}$ is fine enough that $d(r(y), y)<\varepsilon_{1} / 4$ for $y \in Y$. Let $K_{2}$ be the first derived subdivision of $K_{1}$ and $X=\operatorname{St}\left(L_{2}, K_{2}\right)$. This $X$ will satisfy the conclusion of the lemma.

By Lemma 3, there exists $\varepsilon_{2}>0$ such that if $Q$ is any finite $(k+1)$-complex which $\varepsilon_{2}$-deforms to $Y$, then there exists a CW complex $M, Y \subset M \subset R^{2 k+5}$ such that 
(a) $M\left(\varepsilon_{1} / 6\right)$-deforms to $Y$.

(b) $(M, Y)$ has cells only in dimensions $k+1$ and $k+2$.

(c) If $\sigma$ is a cell of $(M, Y)$, then $d(\sigma)<\varepsilon_{1} / 6$.

(d) The Whitehead torsion $\tau(M \cup K, K)=\tau(Q \cup K, K)$.

Let $W=\mathrm{Cm}$ (Int $X, K$ ) and suppose $N$ is a finite complex and $f: K \rightarrow N$ is a homeomorphism such that $f \mid W$ is a p.w.l. map. It will be shown that $f$ is a simple homotopy equivalence. Let $c>0$ and define an embedding $\alpha: N \rightarrow R^{2 k+1} \times R$ by $\alpha(. x)=\left(f^{-1}(x), c\right)$, i.e., $N$ is embedded as a translate of $K$. For simplicity, we disregard $\alpha$ and consider $N$ already embedded in this manner. One important point is that the homeomorphism

$$
f: K \subset R^{2 k+1} \times R \rightarrow N \subset R^{2 k+1} \times R
$$

is now defined by $f(x, 0)=(x, c)$. For later purposes, we consider $K$ and $N \subset R^{2 k+5}$.

Let $h: K \rightarrow N$ be a p.w.l. map approximating $f$ such that $h|W=f| W$. Using Lemma 2 and choosing $c$ small enough, it is a straightforward construction to embed $M_{h}$ in $R^{2 k+5}$ such that

(1) The base of $M_{h}$ is $K$.

(2) The "top" of $M_{h}$ is $N$.

(3) $M_{h}$ contains a subcomplex $Q$ such that $Q \cap K=Y, M_{h}$ collapses to $K \cup Q$, and $Y \cup Q \varepsilon_{2}$-deforms to $Y$ ( $Q$ will be mapping cylinder of $h \mid Y$ ).

The number $\varepsilon_{2}$ was chosen so that there exists a CW complex $M, Y \subset M \subset R^{2 k+5}$, which $\left(\varepsilon_{1} / 6\right)$-deforms to $Y$ and has cells only in dimensions $k+1$ and $k+2$. By Lemma 1, this determines a geometric group associated with $Y$ and an ( $\left.\varepsilon_{1} / 2\right)$-basis. When retracted onto $L$, this gives a geometric group associated with $L$ and an $\varepsilon_{1}$-basis. Using Conjecture $\mathrm{II}_{n}$, the number $\varepsilon_{1}$ was chosen so that this basis determines the 0 element in Wh $\left(\pi_{1}(K)\right)$. This completes the proof.

Conjecture $I_{1}$ and Conjecture $I_{2}$ are true. The purpose of this section is to prove Theorems 1 and 2 below.

THEOREM 1. Conjecture $\mathrm{I}_{1}$ is true.

Proof. Suppose $K$ is a 1-dimensional finite simplicial complex and $\varepsilon>0$. Since each component may be considered independently, suppose that $K$ is connected. Let $K_{1}$ be a subdivision of $K$ such that for each vertex $\alpha$ of $K_{1}, d\left[\operatorname{St}\left(\alpha, K_{1}\right)\right]<\varepsilon / 2$.

Let $K_{2}, K_{3}$, and $K_{4}$ be the second, third, and fourth barycentric subdivisions of $K_{1}$. Let $\alpha_{1}, \alpha_{2}, \ldots, \alpha_{k}$ be the vertices of $K_{1}$ and $\beta_{1}, \beta_{2}, \ldots, \beta_{m}$ be the barycenters of the 1-simplexes of $K_{1}$. Define

$$
\begin{aligned}
U_{i}=\operatorname{St}\left(\alpha_{i}, K_{2}\right), & A_{j}=\operatorname{St}\left(\beta_{j}, K_{4}\right), \\
V_{i}=\operatorname{St}\left(U_{i}, K_{4}\right), & B_{j}=\operatorname{St}\left(A_{j}, K_{4}\right), \\
W_{i}=\operatorname{St}\left(V_{i}, K_{4}\right), & C_{j}=\operatorname{St}\left(B_{j}, K_{4}\right), \\
X_{i}=\operatorname{St}\left(W_{i}, K_{4}\right), & D_{j}=\operatorname{St}\left(C_{j}, K_{4}\right) .
\end{aligned}
$$


If $\beta_{j} \in \mathrm{St}\left(\alpha_{i}, K_{1}\right)$, then $X_{i} \cap A_{j}=$ one point. If $\beta_{j} \notin \operatorname{St}\left(\alpha_{i}, K_{1}\right)$, then $X_{i} \cap A_{j}=\varnothing$. Similar statements hold for $W_{i} \cap B_{j}, V_{i} \cap C_{j}$, and $U_{i} \cap D_{j}$.

Let $\delta>0$ be such that if $L$ is any subcomplex of $K_{4}$, then $V(L, K, \delta)$ $\subset$ Open St $\left(L, K_{4}\right)$. Suppose $G=G(K)$ is a geometric group associated with $K$, $H$ is a free abelian group, and $f: G \rightarrow H$ is a $\delta$-map. The proof consists in showing that this $G, H$, and $\delta$-map $f$ satisfy the conclusion of Conjecture $\mathrm{I}_{1}$.

Let $T_{i, j}=\left\{g \in G\left[\operatorname{Int}\left(W_{i}\right) \cap \operatorname{Int}\left(D_{j}\right)\right]\right.$ : there exists an integer $n \neq 0$ with $f(n g)$ $\left.\in f\left(G\left(V_{i}\right)\right)\right\}$. The basic properties of $T_{i, j}$ are:

(1) $T_{i, j}=0$ when $W_{i} \cap D_{j}=\varnothing$. Thus for a fixed $j$, there are at most two integers $i$ with $T_{i, j} \neq 0$.

(2) The set $T_{i, j}$ is a subgroup of $G\left[\operatorname{Int}\left(W_{i}\right) \cap \operatorname{Int}\left(D_{j}\right)\right]$.

(3) $T_{i, j} \supset G\left[V_{i} \cap \operatorname{Int}\left(D_{j}\right)\right]$.

(4) Any $t \in G\left[\operatorname{Int}\left(W_{i}\right) \cap \operatorname{Int}\left(D_{j}\right)\right]$ can be written uniquely as $t=t_{1}+t_{2}$ where $t_{1} \in G\left[V_{i} \cap \operatorname{Int}\left(D_{j}\right)\right]$ and $t_{2} \in G\left[\operatorname{Int}\left(W_{i}\right) \cap \operatorname{Int}\left(C_{j}\right)\right]$. If $t \in T_{i, j}$ then $t_{1}$ and $t_{2} \in T_{i, j}$. It follows from (3) that any $t_{1} \in G\left[V_{i} \cap \operatorname{Int}\left(D_{j}\right)\right]$ belongs to $T_{i, j}$. Since $T_{i, j}$ is a group, $t_{2} \in T_{i, j}$.

Statement 1. Suppose $V_{i} \cap C_{j} \neq \varnothing, g \in G\left(\operatorname{Int}\left(C_{j}\right)\right)$, and $\exists$ an integer $n \neq 0$ such that $f(n g) \in f\left[G\left(V_{i}\right)+T_{i, j}\right]$. Then $f(g) \in f\left(T_{i, j}\right)$.

Proof. Suppose the hypothesis. Then $\exists v \in G\left(V_{i}\right)$ and $t \in T_{i, j} \ni f(n g)=f(v+t)$. Let $t=t_{1}+t_{2}$ where $t_{1} \in G\left[V_{i} \cap \operatorname{Int}\left(D_{j}\right)\right]$ and $t_{2} \in G\left[\operatorname{Int}\left(W_{i}\right) \cap \operatorname{Int}\left(C_{j}\right)\right]$. Now $t_{1} \in G\left(V_{i}\right)$ and by property (4) above, $t_{2} \in T_{i, j}$. Thus $v$ and $t$ may be chosen so that $v \in G\left(V_{i}\right)$ and $t \in T_{i, j} \cap G\left[\operatorname{Int}\left(W_{i}\right) \cap \operatorname{Int}\left(C_{j}\right)\right]$. Thus $f(v)=f(n g-t)$ where $v \in G\left(V_{i}\right)$ and $(n g-t) \in G\left(\operatorname{Int}\left(C_{j}\right)\right) \subset G\left(\mathrm{Cm}\left(V_{i}\right)\right)$. Therefore by property (2) of a $\delta$-map, $\exists u \in G\left(\operatorname{Int}\left(W_{i}\right) \cap \operatorname{Int}\left(D_{j}\right)\right)$ with $f(u)=f(n g-t)=f(v)$. Since $v \in G\left(V_{i}\right)$, it follows from the definition of $T_{i, j}$ that $u \in T_{i, j}$. Now $f(n g)=f(u+t)$ where $u+t \in T_{i, j}$. Again using the definition of $T_{i, j}, \exists$ an integer $p \neq 0 \ni f(p(u+t))=f(p n g) \in f\left(V_{i}\right)$. Since $\mathrm{Ca}(g) \subset \mathrm{Cm}\left(V_{i}\right)$, it follows from property (2) of a $\delta$-map that $\exists \bar{u} \in$ $G\left(\operatorname{Int}\left(W_{i}\right) \cap \operatorname{Int}\left(D_{j}\right)\right)$ with $f(\bar{u})=f(g)$. Since $f(p n \bar{u})=f(p n g) \in f\left(V_{i}\right)$, it follows that $\bar{u} \in T_{i, j}$. Thus $f(g) \in f\left(T_{i, j}\right)$ and Statement 1 is proved.

Statement 2. Let $j$ be an integer, $1 \leqq j \leqq m$, and $a$ and $b$ be the two integers $\ni W_{a} \cap D_{j} \neq \varnothing$ and $W_{b} \cap D_{j} \neq \varnothing$. Then if $g \in G\left(C_{j}\right)$ and $\exists$ an integer $n \neq 0 \ni f(n g)$ $\in f\left[T_{a, j}+G\left(\mathrm{Cm}\left[\operatorname{Int}\left(D_{j}\right)\right]\right)+T_{b, j}\right]$ then $\exists t_{a} \in T_{a, j}$ and $t_{b} \in T_{b, j}$ with $f\left(t_{a}+t_{b}\right)=f(g)$.

Proof. Let $f(h)=f(n g)$ with $h=h_{1}+h_{2}+h_{3}$ and $g=g_{1}+g_{2}+g_{3}$ where

$$
\begin{array}{ll}
g_{1} \in G\left(\operatorname{Int}\left(W_{a}\right) \cap C_{j}\right), & h_{1} \in T_{a, j}, \\
g_{2} \in G\left(B_{j}\right), & h_{2} \in G\left(\operatorname{Cm}\left[\operatorname{Int}\left(D_{j}\right)\right]\right), \\
g_{3} \in G\left(\operatorname{Int}\left(W_{b}\right) \cap C_{j}\right), & h_{3} \in T_{b, j} .
\end{array}
$$

Now $f\left(n g_{2}\right)=f\left(h-n g_{1}-n g_{3}\right)$ where $n g_{2} \in G\left(B_{j}\right)$ and $\left(h-g n_{1}-n g_{3}\right) \in G\left[\mathrm{Cm}\left(B_{j}\right)\right]$. Thus by property (2) of a $\delta$-map, $\exists u \in G\left[C_{j} \cap X_{a}\right], v \in G\left[C_{j} \cap X_{b}\right]$ with $f(u+v)$ $=f\left(g_{2}\right)$. 
Furthermore $f\left(h_{2}\right)=f\left(n g-h_{1}-h_{3}\right)$ where $h_{2} \in G\left[\operatorname{Cm}\left(\operatorname{Int} D_{j}\right)\right],\left(n g-h_{1}-h_{3}\right)$ $\in G\left[\right.$ Int $D_{j}$ ]. Again by property (2) of a $\delta$-map, $\exists x \in G\left(V_{a}\right), y \in G\left(V_{b}\right)$ with $f(x+y)$ $=f\left(h_{2}\right)$.

Collecting the above results, $f\left(n\left[g_{1}+u+v+g_{3}\right]\right)=f\left(h_{1}+x+y+h_{3}\right)$ or

$$
f\left(n\left[g_{1}+u\right]-\left[h_{1}+x\right]\right)=f\left(\left[y+h_{3}\right]-n\left[v+g_{3}\right]\right)
$$

where $g_{1}, u, h_{1}, x \in G\left(X_{a}\right)$ and $y, h_{3}, v, g_{3} \in G\left(X_{b}\right)$. Since the distance from $X_{a}$ to $X_{b}$ is greater than $\delta$, it follows from property (1) of $\delta$-map that $f\left[G\left(X_{a}\right)\right] \cap f\left[G\left(X_{b}\right)\right]=0$ and thus $f\left(n\left[g_{1}+u\right]\right)=f\left(h_{1}+x\right)$ and $f\left(n\left[v+g_{3}\right]\right)=f\left(y+h_{3}\right)$. Now $\left[g_{1}+u\right] \in G\left(C_{j}\right)$ and $\left[h_{1}+x\right] \in T_{a, j}+G\left(V_{a}\right)$ and it follows from Statement 1 that $\exists t_{a} \in T_{a, j} \ni f\left(t_{a}\right)$ $=f\left(g_{1}+u\right)$. Similarly, $\exists t_{b} \in T_{b, j} \ni f\left(t_{b}\right)=f\left(v+g_{3}\right)$. Therefore

$$
f\left(t_{a}+t_{b}\right)=f\left(g_{1}+u+v+g_{3}\right)=f\left(g_{1}+g_{2}+g_{3}\right)=f(g) .
$$

This proves Statement 2 .

For any given $i, 1 \leqq i \leqq k$, define $G_{i}^{\prime}$ by $G_{i}^{\prime}=G\left(U_{i}\right) \oplus\left[\oplus_{(i, j) \in \alpha} T_{i, j}\right]$ where $\alpha$ $=\left\{(i, j): U_{i} \cap D_{j} \neq \varnothing\right\}$. Then $G\left(U_{i}\right) \subset G\left(V_{i}\right) \subset G_{i}^{\prime} \subset G\left(W_{i}\right)$ and $G_{i}^{\prime} \cap\left[+_{j \neq i} G_{j}^{\prime}\right]=0$. Let $G^{\prime}=\bigoplus_{i=1}^{k} G_{i}^{\prime}$.

STATEMENT 3. $H / f\left(G^{\prime}\right)$ has no torsion.

Proof. Suppose $g \in G$ and for some integer $n>1, f(n g) \in f\left(G^{\prime}\right)$. Show $f(g)$ $\in f\left(G^{\prime}\right)$. Now $g=h^{\prime}+g_{1}+g_{2}+\cdots+g_{m}$ where $h^{\prime} \in \bigoplus_{i=1}^{k} G\left(V_{i}\right) \subset G^{\prime}$ and $g_{j} \in G\left(C_{j}\right)$. Thus. $f\left[n\left(g_{1}+g_{2}+\cdots+g_{m}\right)\right] \in f\left(G^{\prime}\right)$ and it must be shown that $f\left(g_{1}+g_{2}+\cdots+g_{m}\right)$ $\in f\left(G^{\prime}\right)$. Since $f(n g) \in f\left(G^{\prime}\right)$ and $f\left(n h^{\prime}\right) \in f\left(G^{\prime}\right), \exists g^{\prime} \in G^{\prime}$ with

$$
f\left(n g_{1}\right)=f\left(g^{\prime}-n\left(g_{2}+g_{3}+\cdots+g_{m}\right)\right) .
$$

It now follows from Statement 2 that $f\left(g_{1}\right) \in f\left(G^{\prime}\right)$. Similarly, $f\left(g_{j}\right) \in f\left(G^{\prime}\right)$, and therefore $f\left(g_{1}+g_{2}+\cdots+g_{m}\right) \in f\left(G^{\prime}\right)$.

Statement 4. Let $\pi: H \rightarrow H \mid f\left(G^{\prime}\right)$ be the projection. Then

$$
H \mid f\left(G^{\prime}\right)=\bigoplus_{j=1}^{m} \pi f\left[G\left(C_{j}\right)\right] .
$$

Proof. It must be shown, for example, that $\pi f\left[G\left(C_{1}\right)\right] \cap\left(+_{j=2}^{m} \pi f\left[G\left(C_{j}\right)\right]\right)=0$. Suppose $\pi f\left(c_{1}\right)=\pi f\left(c_{2}+c_{3}+\cdots+c_{m}\right)$ where $c_{j} \in G\left(C_{j}\right)$. This means $\exists g^{\prime} \in G^{\prime}$ with $f\left(c_{1}\right)=f\left(c_{2}+\cdots+c_{m}+g^{\prime}\right)$. Therefore by Statement $2 f\left(c_{1}\right) \in f\left(G^{\prime}\right)$ and $\pi f\left(c_{1}\right)=0$. This proves Statement 4 .

Now $f\left(G_{i}^{\prime}\right)$ is a subgroup of the free abelian group $H$ and thus $f\left(G_{i}^{\prime}\right)$ is free abelian. Let $F_{i}$ be a finite subset of $G_{i}^{\prime} \ni f\left(F_{i}\right)$ is a free abelian basis of $f\left(G_{i}^{\prime}\right)$. According to Statement 3,H/f(G') is a free abelian group and according to Statement 4, $H \mid f\left(G^{\prime}\right)=\oplus \pi f\left[G\left(C_{j}\right)\right]$. Let $E_{j}$ be a finite subset of $G\left(C_{j}\right) \ni \pi f\left(E_{j}\right)$ is a free basis for $\pi f\left[G\left(C_{j}\right)\right]$.

STATEMENT 5. The set $f\left[\left(\cup E_{j}\right) \cup\left(\cup F_{i}\right)\right]$ is a free basis for $H$ satisfying the conclusion of the theorem. 
Proof. The fact that it is a free basis follows from the exact sequence $0 \rightarrow f\left(G^{\prime}\right)$ $\rightarrow H \rightarrow H \mid f\left(G^{\prime}\right) \rightarrow 0$ and the fact that $f\left(\bigcup F_{i}\right)$ is a free basis for $f\left(G^{\prime}\right)$ and $\pi f\left(\bigcup E_{j}\right)$ is a free basis for $H \mid f\left(G^{\prime}\right)$.

If $g \in\left(\bigcup E_{j}\right)$ or $g \in\left(\bigcup F_{i}\right)$ then $d[\mathrm{Ca}(g)]<\varepsilon$ because, for some vertex $\alpha_{t}$, $\mathrm{Ca}(g) \subset \mathrm{St}\left(\alpha_{t}, K_{1}\right)$ and $d\left[\mathrm{St}\left(\alpha_{t}, K_{1}\right)\right]<\varepsilon / 2<\varepsilon$.

It remains to be shown that if $S \subset K$, then $f[G(S)] \subset$ the subgroup of $H$ generated by $f(R)$ where $R=\left\{c \in\left(\bigcup E_{j}\right) \cup\left(\bigcup F_{i}\right): \mathrm{Ca}(c) \subset V(S, \varepsilon)\right\}$. Now any $g \in G(S)$ can be written as $g=g_{1}+g_{2}+\cdots+g_{t}$ where $g_{v} \in\left[\right.$ some $\left.G\left(C_{j}\right)\right] \cap G(S)$ or $g_{v} \in$ [some $G\left(\right.$ Int $\left.\left.V_{i}\right)\right] \cap G(S) \subset$ [some $\left.G_{i}^{\prime}\right] \cap G(S)$. It will be shown that each $g_{v} \in$ the subgroup of $H$ generated by $f(R)$ and the theorem will follow.

Suppose first that $g_{v} \in G\left(\operatorname{Int} V_{i}\right) \cap G(S)$. Now $S \cap\left(\right.$ Int $\left.V_{i}\right) \neq \varnothing$ (assuming $\left.g_{v} \neq 0\right)$. Since $d\left[\operatorname{St}\left(\alpha_{i}, K_{1}\right)\right]<\varepsilon / 2$, it follows that $V(S, \varepsilon) \supset \mathrm{St}\left(\alpha_{i}, K_{1}\right) \supset \mathrm{Ca}\left(G_{i}^{\prime}\right)$ $\supset \mathrm{Ca}\left(F_{i}\right)$. Therefore $F_{i} \subset R$ and the result follows because $f\left(g_{v}\right) \in$ the subgroup generated by $f\left(F_{i}\right)$.

For the other case, suppose $g_{v} \in G\left(C_{j}\right) \cap G(S)$. Then $\pi f\left(g_{v}\right)=\pi f\left(n_{1} e_{1}+\cdots+n_{r} e_{r}\right)$ where each $e_{u} \in E_{j}$. Then $f\left(g_{v}-\left[n_{1} e_{1}+\cdots+n_{r} e_{r}\right]\right) \in f\left(G^{\prime}\right)$. Since $\left(g_{v}-\left[n_{1} e_{1}+\cdots\right.\right.$ $\left.\left.+n_{r} e_{r}\right]\right) \in G\left(C_{j}\right)$, it follows from Statement 2 that $\exists t_{a} \in T_{a, j}$ and $t_{b} \in T_{b, j} \ni f\left(t_{a}+t_{b}\right)$ $=f\left(g_{v}-\left[n_{1} e_{1}+\cdots+n_{r} e_{r}\right]\right)$. Now $f\left(t_{a}\right)$ and $f\left(t_{b}\right)$ are in the subgroups generated by $f\left(F_{a}\right)$ and $f\left(F_{b}\right)$ respectively. Thus $f\left(g_{v}\right)$ is in the subgroup generated by $f\left(F_{a} \cup F_{b} \cup E_{j}\right)$. Since $\mathrm{Ca}\left(E_{a} \cup E_{b} \cup E_{j}\right) \subset V\left(\mathrm{Ca}\left(g_{v}\right), \varepsilon\right)$, this completes the proof of Statement 5 and the theorem.

THEOREM 2. Conjecture $\mathrm{I}_{n} \Rightarrow$ Conjecture $\mathrm{II}_{n+1}$ for $n=0,1,2, \ldots$

Sketch of proof. Since the proof is hidden in a mass of details, a crude sketch of the ideas involved is presented. Suppose Conj. $\mathrm{I}_{n}$ and Conj. $\mathrm{II}_{n}$ are true and show Conj. $\mathrm{II}_{n+1}$ is true. Consider the simplest case possible-an $(n+1)$-simplex $\sigma$ with $d(\sigma)<\varepsilon$. Suppose $G(\sigma)$ is a geometric group with a $\delta$-basis $\left\{a_{i}\right\}_{i=1}^{v}$. Let Bdry $\sigma \subset \Delta_{1}$ $\subset \Delta_{2} \subset \sigma$ where $\Delta_{2}$ retracts to Bdry $\sigma$. Define $A=\left\{a_{i}: a_{i} \in G\left(\Delta_{2}\right)\right\}$. The basic step in the proof is showing that $\pi: G\left(\Delta_{2}\right) \rightarrow G\left(\Delta_{2}\right) / A$ is a $\delta_{1}$-map (actually $\Delta_{2}$ must be retracted onto Bdry $\sigma$ so that the inductive hypothesis will apply-however at this point we simply think of $\Delta_{2}$ as being $n$-dimensional). Applying Conj. $\mathrm{I}_{n}$ to the map $\pi$ yields an extension of $A$ to a $\delta_{2}$-basis of $G\left(\Delta_{3}\right)$. The transformation (c) determined by this basis is the product of $\varepsilon$-blocked matrices (Conj. $\mathrm{II}_{n}$ ) and $(c)$ agrees with $\left(a_{i, j}\right)$ on $G\left(\Delta_{1}\right)$. This means that if $\left(a_{i, j}\right)=(d)(c)$, then $(d)$ is the identity on $G\left(\Delta_{1}\right)$ and thus $\varepsilon$-blocked. In the general case the complex will have $(n+1)$ simplexes $\sigma_{1}, \sigma_{2}, \ldots, \sigma_{r}$. As before, $(d)$ will be the identity on some neighborhood $\Delta_{1}$ of the $n$-skeleton and will carry each $G\left(\sigma_{i}\right)$ into itself. Thus $(d)$ will still be $\varepsilon$ blocked.

Proof. The proof is by induction on $n$. Suppose Conj. $\mathrm{I}_{n}$ is true for some $n \geqq 0$. Suppose by induction that Conj. $\mathrm{II}_{n}$ is true and show that Conj. $\mathrm{II}_{n+1}$ is true. Suppose that $K$ is a finite simplicial complex with $\operatorname{dim} K=n+1$ and that $\varepsilon>0$. Let $K_{1}$ be a subdivision of $K$ with $d\left[\operatorname{St}\left(\alpha, K_{1}\right)\right]<\varepsilon$ for each vertex $\alpha$ of $K_{1}$. Let $K_{1}^{n}$ be the 
$n$-skeleton of $K_{1}, 0$ an open set, $K_{1}^{n} \subset 0 \subset K$ and $r: 0 \rightarrow K_{1}^{n}$ a retraction. Let $\delta_{2}>0$ be such that Conj. II holds true for the complex $K_{1}^{n}$ and the positive number $\varepsilon / 2$. Let $\delta_{1}>0$ be such that Conj. I holds true for the complex $K_{1}^{n}$ and the positive number $\delta_{2} / 8$. Let $\Delta_{1}, \Delta_{2}$ be closed sets with $K_{1}^{n} \subset \operatorname{Int} \Delta_{1}, \Delta_{1} \subset \operatorname{Int} \Delta_{2}$, and $d(x, r(x))<$ $\min \left(\varepsilon / 4, \delta_{2} / 16, \delta_{1} / 8\right)$ for all $x \in \Delta_{2}$. Let $\delta>0$ be such that $\delta<\min \left(\delta_{2} / 4, \delta_{1} / 4\right)$ and $V\left(K_{1}, K, \delta\right) \subset$ Int $\Delta_{1}$, and $V\left(\Delta_{1}, K, \delta\right) \subset$ Int $\Delta_{2}$. It will be shown that this $\delta$ satisfies the conclusion of Conj. II for the complex $K$ and the positive number $\varepsilon$. Let $\left\{a_{i}\right\}_{i=1}^{v}$ be any $\delta$-basis for $G$. Since the conclusion of Conj. II allows relabeling this basis, it will be assumed that $p_{i} \in \mathrm{Ca}\left(a_{i}\right)$ [see proof of Proposition 0, part (b)].

Let $A \subset G$ be the subgroup generated by $\left\{a_{i}: \mathrm{Ca}\left(a_{i}\right) \subset \Delta_{2}\right\}$ and $\pi: G \rightarrow G / A$ be the projection.

STATEMENT 1. $G\left(\Delta_{2}\right) / A$ has no torsion and is thus a free abelain group.

This follows from the fact that $G / A$ has no torsion because $A$ is generated by a subset of a free basis.

Statement 2. If $g_{1}, g_{2} \in G\left(\Delta_{2}\right)$ and $V\left(\mathrm{Ca}\left(g_{1}\right), K, \delta\right) \cap V\left(\mathrm{Ca}\left(g_{2}\right), K, \delta\right)=\varnothing$, then $\pi\left(g_{1}\right)=\pi\left(g_{2}\right) \Rightarrow \pi\left(g_{1}\right)=0$.

Proof. Suppose $\pi\left(g_{1}\right)=\pi\left(g_{2}\right)$. Then $g_{1}=g_{2}+n_{1} a_{1}+\cdots+n_{v} a_{v}$ where $n_{i} \neq 0 \Rightarrow$ $a_{i} \in A$. Due to property (2) of the $\delta$-basis $\left\{a_{i}\right\}, g_{1}=g_{1,1} a_{1}+\cdots+g_{v, 1} a_{v}$ where $g_{i, 1} \neq 0 \Rightarrow \mathrm{Ca}\left(a_{i}\right) \subset V\left(\mathrm{Ca}\left(g_{1}\right), K, \delta\right)$ and $g_{2}=g_{1,2} a_{1}+\cdots+g_{v, 2} a_{v}$ where $g_{i, 2} \neq 0 \Rightarrow$ $\mathrm{Ca}\left(a_{i}\right) \subset V\left(\mathrm{Ca}\left(g_{2}\right), K, \delta\right)$. Since the $a_{i}$ form a free basis and $g_{1,1} a_{1}+\cdots$ $+g_{v, 1} a_{v}=g_{1,2} a_{1}+\cdots+g_{v, 2} a_{v}+n_{1} a_{1}+\cdots+n_{v} a_{v}$ the coefficients must be the same on each side of the equation. Since $V\left(\mathrm{Ca}\left(g_{1}\right), K, \delta\right) \cap V\left(\mathrm{Ca}\left(g_{2}\right), K, \delta\right)=\varnothing$, $g_{i, 1}=g_{i, 2} \Rightarrow g_{i, 1}=0$. Now $a_{i} \notin A \Rightarrow n_{i}=0 \Rightarrow g_{i, 1}=g_{i, 2} \Rightarrow g_{i, 1}=g_{i, 2}=0$. Therefore $g_{1}$ and $g_{2} \in A$ and $\pi\left(g_{1}\right)=\pi\left(g_{2}\right)=0$.

StATEMEnT 3. Suppose $S_{1} \cup S_{2}=\Delta_{2}$ and $S_{1} \cap S_{2}=\varnothing$. If $g \in G\left(S_{2}\right)$ and $\exists$ an integer $n \neq 0$ such that $\pi(n g) \in \pi\left[G\left(S_{1}\right)\right]$, then $\exists \bar{g} \in G\left[V\left(S_{1}, \Delta_{2}, \delta\right) \cap V\left(S_{2}, \Delta_{2}, \delta\right)\right]$ with $\pi(\bar{g})=\pi(g)$.

Proof. Suppose $h \in G\left(S_{1}\right)$ and $\pi(n g)=\pi(h)$. Then $n g=h+n_{1} a_{1}+\cdots+n_{v} a_{v}$ where $n_{i} \neq 0 \Rightarrow a_{i} \in A$. Also $g=c_{1} a_{1}+\cdots+c_{v} a_{v}$ where $c_{i} \neq 0 \Rightarrow \mathrm{Ca}\left(a_{i}\right) \subset V\left(S_{2}, K, \delta\right)$ and $h=d_{1} a_{1}+\cdots+d_{v} a_{v}$ where $d_{i} \neq 0 \Rightarrow \mathrm{Ca}\left(a_{i}\right) \subset V\left(S_{1}, K, \delta\right)$. Write

$$
g=\left(\underset{i \in \alpha}{+} c_{i} a_{i}\right)+\left(\underset{j \in \beta}{+} c_{j} a_{j}\right)
$$

where

$$
\begin{aligned}
& \alpha=\left\{j: 1 \leqq i \leqq v, c_{i} \neq 0, \text { and } \mathrm{Ca}\left(a_{i}\right) \subset V\left(S_{1}, K, \delta\right)\right\} \\
& \beta=\left\{j: 1 \leqq j \leqq v, c_{j} \neq 0, \text { and } \mathrm{Ca}\left(a_{j}\right) \notin V\left(S_{1}, K, \delta\right)\right\}
\end{aligned}
$$

From the equation

$$
n\left(c_{1} a_{1}+\cdots+c_{v} a_{v}\right)=d_{1} a_{1}+\cdots+d_{v} a_{v}+n_{1} a_{1}+\cdots+n_{v} a_{v}
$$


and the fact that $\mathrm{Ca}\left(a_{i}\right) \notin V\left(S_{1}, K, \delta\right) \Rightarrow d_{i}=0$, it follows that for each $j \in \beta$, $n c_{j}=n_{j}$. Thus for each $j \in \beta, n_{j} \neq 0$ and $a_{j} \in A$. Therefore $\pi\left(+_{j \in \beta} c_{j} a_{j}\right)=0$ and $\pi\left(+_{i \in \alpha} c_{i} a_{i}\right)=\pi(g)$. Now write $\left(+_{i \in \alpha} c_{i} a_{i}\right)=\bar{g}+g^{\prime}$ where

$$
\begin{aligned}
\mathrm{Ca}(\bar{g}) & \subset\left[V\left(S_{1}, K, \delta\right) \cap \Delta_{2}\right]=V\left(S_{1}, \Delta_{2}, \delta\right), \\
\mathrm{Ca}\left(g^{\prime}\right) & \subset\left[V\left(S_{1}, K, \delta\right) \cap \mathrm{Cm}\left(\Delta_{2}\right)\right] \subset \mathrm{Cm}\left(\Delta_{2}\right) .
\end{aligned}
$$

With this notation, $\pi\left(g^{\prime}\right)=\pi(g-\bar{g})$, i.e., $g^{\prime}=g-\bar{g}+a$ where $a \in A$. But $(g-\bar{g}+a)$ $\in G\left(\Delta_{2}\right)$ and therefore $g^{\prime}=0$. This gives $\pi(\bar{g})=\pi(g)$ where $\mathrm{Ca}(\bar{g}) \subset V\left(S_{1}, \Delta_{2}, \delta\right)$ and $\mathrm{Ca}(\bar{g}) \subset V(\mathrm{Ca}(g), K, \delta) \subset V\left(S_{2}, K, \delta\right)$. Since $\mathrm{Ca}(\bar{g}) \subset \Delta_{2}$, the second inclusion may be written $\mathrm{Ca}(\bar{g}) \subset V\left(S_{2}, \Delta_{2}, \delta\right)$. This proves Statement 3.

Now summarizing the proof so far:

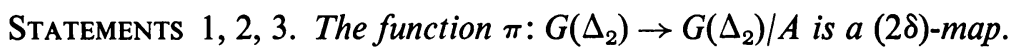

Now consider the geometric group $G^{\prime}$ associated with the complex $K_{1}^{n}$ and generated by the symbols $r\left(p_{i}\right)$ where $p_{i} \in \Delta_{2}$. Even though $r\left(p_{i}\right)$ and $r\left(p_{j}\right)$ may represent the same point of $K_{1}^{n}$, they are distinct free generators of $G^{\prime}$ whenever $i \neq j$. Since $p_{i}$ is a point of a complex and also an element of a group, the function $r$ can also be considered as a retraction and a group homomorphism $r: G\left(\Delta_{2}\right) \rightarrow G^{\prime}$. This is done by defining $r\left(n_{1} p_{1}+\cdots+n_{v} p_{v}\right)=n_{1} r\left(p_{1}\right)+n_{2} r\left(p_{2}\right)+\cdots+n_{v} r\left(p_{v}\right)$ whenever $n_{1} p_{1}+\cdots+n_{v} p_{v} \in G\left(\Delta_{2}\right)$. Then $r$ is an isomorphism, $r: G\left(\Delta_{2}\right) \approx G^{\prime}$ and $A^{\prime}$ is defined by $A^{\prime}=r(A)$. The projection map $\pi^{\prime}: G^{\prime} \rightarrow G^{\prime} \mid A^{\prime}$ is given by $\pi^{\prime}=r \pi r^{-1}$.

StATEMENT $1^{\prime}, G^{\prime} \mid A^{\prime}$ has no torsion and is thus a free abelian group.

This follows from Statement 1 and the fact that $G\left(\Delta_{2}\right) / A \approx G^{\prime} \mid A^{\prime}$.

Statement $2^{\prime}$. If $g_{1}, g_{2} \in G^{\prime}$ and $V\left(\mathrm{Ca}\left(g_{1}\right), K_{1}^{n}, \delta_{1} / 2\right) \cap V\left[\mathrm{Ca}\left(g_{2}\right), K_{1}^{n}, \delta_{1} / 2\right]=\varnothing$ then $\pi^{\prime}\left(g_{1}\right)=\pi^{\prime}\left(g_{2}\right) \Rightarrow \pi^{\prime}\left(g_{1}\right)=0$.

Statement $3^{\prime}$. Suppose $S_{1} \cup S_{2}=K_{1}^{n}$ and $S_{1} \cap S_{2}=\varnothing$. If $g \in G^{\prime}\left(S_{2}\right)$ and $\exists$ an integer $n \neq 0$ such that $\pi(n g) \in \pi\left[G^{\prime}\left(S_{1}\right)\right]$, then $\exists \bar{g} \in G\left[V\left(S_{1}, K_{1}^{n}, \delta_{1}\right) \cap V\left(S_{2}, K_{1}^{n}, \delta_{1}\right)\right]$ with $\pi^{\prime}(\bar{g})=\pi^{\prime}(g)$.

Statements $1^{\prime}, 2^{\prime}, 3^{\prime}$. The function $\pi^{\prime}: G^{\prime} \rightarrow G^{\prime} \mid A^{\prime}$ is a $\delta_{1}$-map.

The proofs of Statements $2^{\prime}$ and $3^{\prime}$ follow immediately from Statements 2 and 3 and the facts that $d(x, r(x))<\delta_{1} / 8$ and $\delta<\delta_{1} / 4$. Now in order to simplify the proof and keep the notation under control, we suppose that there is only one $(n+1)$ simplex $\sigma$ in $K_{1}$. The general case is no different except each $(n+1)$-simplex must be handled independently.

STATEMENT $4^{\prime} . \exists$ a collection $\left\{d_{1}^{\prime}, d_{2}^{\prime}, \ldots, d_{s}^{\prime}\right\} \subset G^{\prime}$ such that:

(a) $\left\{\pi^{\prime}\left(d_{i}^{\prime}\right)\right\}_{i=1}^{s}$ is a free basis for $G^{\prime} \mid A^{\prime}$.

(b) $d\left(\mathrm{Ca}\left(d_{i}^{\prime}\right)\right)<\delta_{2} / 8$ for $i=1,2, \ldots, s$.

(c) If $S \subset K_{1}^{n}$, then $\pi^{\prime}\left(G^{\prime}(S)\right) \subset$ the subgroup of $H$ generated by

$$
\left\{\pi^{\prime}\left(d_{i}^{\prime}\right): \mathrm{Ca}\left(d_{i}^{\prime}\right) \subset V\left(S, K_{1}^{n}, \delta_{2} / 8\right)\right\} .
$$


The collection $\left\{d_{1}^{\prime}, d_{2}^{\prime}, \ldots, d_{s}^{\prime}\right\} \cup\left\{r\left(a_{i}\right): a_{i} \in A\right\}$ is a $\delta_{2}$-basis for $G^{\prime}$.

Proof. By assumption, Conjecture $I$ is true for $n$-dimensional complexes. The number $\delta_{1}$ was chosen so that the conclusion of Conj. I holds for the positive number $\delta_{2} / 8$, the complex $K_{1}^{n}$, and $\delta_{1}$-maps from geometric groups associated with $K_{1}^{n}$. Statement $4^{\prime}$ is the conclusion of Conj. I applied to the $\delta_{1}$-map $\pi^{\prime}: G^{\prime} \rightarrow G^{\prime} \mid A^{\prime}$. The fact that the collection $\left\{d_{1}^{\prime}, d_{2}^{\prime}, \ldots, d_{s}^{\prime}\right\} \cup\left\{r\left(a_{i}\right): a_{i} \in A\right\}$ is a $\delta_{2}$-basis for $G^{\prime}$ follows immediately from (b), (c), the fact that $\left\{a_{i}\right\}$ forms a $\left(\delta_{2} / 4\right)$-basis for $G$, and the fact that $d(r(x), x)<\delta_{2} / 16$ for all $x \in \Delta_{2}$.

StATEMENT $5^{\prime}$. Given any ordering of $\left\{r\left(p_{i}\right): p_{i} \in G\left(\Delta_{3}\right)\right\}, \exists$ an ordering of the $\delta_{2}$-basis $\left\{d_{1}^{\prime}, d_{2}^{\prime}, \ldots, d_{s}^{\prime}\right\} \cup\left\{r\left(a_{i}\right): a_{i} \in A\right\}$ such that the automorphism $T^{\prime}$ which sends the first basis onto the second, is the product of $(n+1)(\varepsilon / 2)$-blocked automorphisms: $T^{\prime}=T_{n+1}^{\prime} T_{n}^{\prime} \cdots T_{1}^{\prime}$.

Proof. The number $\delta_{2}$ was chosen so that the conclusion of Conj. II holds for the complex $K_{1}^{n}$ and the positive number $\varepsilon / 2$. Statement $5^{\prime}$ is simply the conclusion of Conj. II.

Now define $d_{i} \in G\left(\Delta_{3}\right)$ by $d_{i}=r^{-1}\left(d_{i}^{\prime}\right)$. Since $A$ generates $G\left(\Delta_{1}\right)$ and $\left\{\pi\left(d_{i}\right)\right\}$ generates $G\left(\Delta_{2}\right) / A$, the $d_{i}$ may be restricted to $G\left(\Delta_{2}-\Delta_{1}\right)$, i.e., assume $d_{i} \in G\left(\Delta_{2}-\Delta_{1}\right)$. Let $T=r^{-1} T^{\prime} r$ and $T_{i}=r^{-1} T_{i}^{\prime} r$.

STATEMENTs 4, 5. Summary of the proof to this point. Given any ordering of $\left\{p_{i}: p_{i} \in G\left(\Delta_{3}\right)\right\}, \exists$ an ordering of the basis $\left\{d_{1}, d_{2}, \ldots, d_{s}\right\} \cup\left\{a_{i}: a_{i} \in A\right\}$ such that the automorphism $T: G\left(\Delta_{2}\right) \rightarrow G\left(\Delta_{2}\right)$ which sends the first basis to the second, is the product of $(n+1) \varepsilon$-blocked automorphisms: $T=T_{n+1} T_{n} \cdots T_{1}$.

Proof of Statements 4, 5. If $S \subset K_{1}^{n}$ and $d(S)<\varepsilon / 2$, then $d\left[r^{-1}(S) \cap \Delta_{2}\right]<\varepsilon$, because $d(r(x), x)<\varepsilon / 4$ for all $x \in \Delta_{2}$. The $T_{i}$ are $\varepsilon$-blocked because the $T_{i}^{\prime}$ are $(\varepsilon / 2)$-blocked. Thus the proof follows from Statements $4^{\prime}, 5^{\prime}$.

Extend $T$ and $T_{i}$ to all of $G$ by defining $T\left|G\left[\mathrm{Cm}\left(\Delta_{2}\right)\right]=T_{i}\right| G\left[\mathrm{Cm}\left(\Delta_{2}\right)\right]=$ the identity, and note that $T_{i}$ is still $\varepsilon$-blocked. Let $(c)$ be the matrix which represents $T$ under the natural ordering of $\left\{p_{i}\right\}_{i=1}^{v}$. If $a_{t} \in G\left(\Delta_{2}\right)$, it is in the basis which determined $T$, i.e., $\exists p_{r}$ with $(c) p_{r}=a_{t}$. Therefore, $\exists$ a matrix $(Y)$ obtained from the identity by interchanging columns, such that $(c)(Y) p_{t}=a_{t}$ for each $t$ with $a_{t} \in G\left(\Delta_{2}\right)$. Let $(d)$ be the unique matrix such that $\left(a_{i, j}\right)=(d)(c)(Y)$.

STATEMENT 6. $(d) a_{t}=a_{t}$ for all $a_{t} \in G\left(\Delta_{2}\right)$.

Proof. $\left(a_{i, j}\right) p_{t}=a_{t}=(c)(Y) p_{t}$ for all $t$ with $a_{t} \in G\left(\Delta_{2}\right)$.

STATEMENT 7. $d\left(p_{t}\right)=p_{t}$ for all $p_{t} \in G\left(\Delta_{1}\right)$.

Proof. This follows from Statement 6 and the fact that $\left\{a_{i}: a_{i} \in G\left(\Delta_{2}\right)\right\}$ generates $G\left(\Delta_{1}\right)$. This shows that the matrix $(d)$ is $\varepsilon$-blocked and since $(c)$ is the product of $(n+1) \varepsilon$-blocked matrices, and $\left(a_{i, j}\right)(Y)^{-1}=(d)(c)$, the proof is complete.

COROLlary 1. Suppose $K$ is a finite connected complex and $L$ is a connected subcomplex with $\operatorname{dim} L \leqq 2$. Then $\exists$ a subcomplex $X$ of some subdivision of $K$, 
$L \subset$ Int $(X, K)$, such that if $N$ is any complex and $f: K \rightarrow N$ is any homeomorphism with $f \mid \mathrm{Cm}$ (Int $[X, K], K)$ p.w.l., then $f$ is a simple homotopy equicalence.

Proof. The proof is immediate from Lemma 5 and the fact that Conj. $\mathrm{II}_{2}$ is true. (The hypothesis that $L$ be connected was merely a convenience-Lemma 5 and Corollary 1 are true without this restriction.)

Corollary 2. Suppose $M$ is a p.w.l. n-manifold and $K \subset M$ is a connected 2-complex topologically embedded in $M$. Then $\exists \delta>0$ such that if $U$ is any complex topologically embedded in $M, K \subset U \subset M, K$ is a subcomplex of $U$, and $U \delta$-deforms to $K$, then $K \subset U$ is a simple homotopy equivalence. If in addition $K$ and $U$ are subcomplexes of $M, U$ is an $n$-manifold with boundary, $K \subset \operatorname{Int}(U)$, and $n \geqq 6$, then $U$ is a regular neighborhood of $K$.

Proof. It follows from Lemmas 3 and 4 that $\exists \delta>0$ such that if $U \delta$-deforms to $K$, then $K \subset U$ is a simple homotopy equivalence. Suppose $K$ and $U$ are subcomplexes of $M, U$ is an $n$-manifold with boundary, $K \subset \operatorname{Int}(U)$, and $n \geqq 6$. Let $U_{1}$ be a regular neighborhood of $K, U_{1} \subset$ Int $U$. Then $U$-Int $\left(U_{1}\right)$ is an $H$-cobordism. Since $K \subset U_{1}$ and $K \subset U$ are simple homotopy equivalences, and $\pi_{1}\left(U_{1}\right) \approx \pi_{1}\left(\partial U_{1}\right)$, it follows that $\partial U_{1} \subset U-\operatorname{Int}\left(U_{1}\right)$ is a simple homotopy equivalence. Therefore $U-\operatorname{Int}\left(U_{1}\right)$ is p.w.l. homeomorphic to $\partial U_{1} \times I([10]$, [11], [12]). Thus $U$ is a regular neighborhood of $K$.

DEFINITION. If $K$ is a finite connected complex, an abstract regular neighborhood $X^{n}$ of $K$ is a compact p.w.l. manifold containing $K$ as a subcomplex such that $X$ collapses to $K$.

Corollary 3. Suppose $K$ is a finite, connected 2-complex and $X^{n}$ is an abstract regular neighborhood of $K, n \geqq 6$, and $Y$ is a p.w.l. manifold and $f: X \rightarrow Y$ is a homeomorphism onto. Then $f$ is a simple homotopy equivalence.

Proof. Since $n \geqq 6, f$ is isotopic $(\bmod \partial X)$ to a homeomorphism which, when restricted to $K$ is a p.w.l. embedding ([18] or [19]). Thus suppose $f$ itself has this property. Now $\tau(f)=\tau(i)$ where $i: f(K) \rightarrow Y$ is the inclusion. Let $\delta>0$ satisfy the conclusion of Corollary 2 for the complex $f(K)$. Since $Y$ is homeomorphic to $X, \exists$ a topological embedding $h: Y \rightarrow Y$ such that

(1) $h$ is topologically isotopic to Id: $Y \rightarrow Y$,

(2) $h \mid f(K)=\mathrm{Id}: f(K) \rightarrow f(K)$, and

(3) $f(K)$ is a $\delta$-deformation retract of $h(Y)$. Now by Corollary 2, j: $f(K) \rightarrow h(Y)$ $=U$ is a simple homotopy equivalence. Since $\tau(f)=\tau(i)=\tau(j)=0$, this completes the proof.

Farrell and Hsiang have constructed $h$-cobordisms which are not topologically trivial [20]. It follows from the next corollary, that if $\pi$ is a finitely presented group and $\mathrm{Wh}(\pi) \neq 0$, then $\exists$ a p.w.l. $h$-cobordism $W$ with $\pi_{1}(W)=\pi$ and such that $W$ is not topologically trivial. This result has also been obtained by A. Casson. 
Corollary 4. Suppose $K$ is a finite connected 2-complex and $X^{n}$ is an abstract regular neighborhood of $K, n \geqq 6$. If $W$ is a p.w.l. h-cobordism, one of whose boundary components is $\partial X$, then $W$ is homeomorphic to $\partial X \times I$ iff $\tau(W, \partial X)=0$.

Proof. If $\tau(W, \partial X)=0$, then $W$ is p.w.l. homeomorphic to $\partial X \times I$ by p.w.l. Smale theory. Suppose $W$ is homeomorphic to $\partial X \times I$. If $Y=W \cup X$ identified by $\partial X$, then $\exists$ a homeomorphism $f: X \rightarrow Y$ which is homotopic to Id: $X \rightarrow X$. By Corollary $3, \tau(f)=0$. Since $\tau(W, \partial X)=\tau(Y, X)=\tau(f)=0$, the proof is complete.

Appendix. Geometric groups are related to several problems in topology, and some of these are mentioned here. No proofs are presented but brief sketches of the ideas are included.

\section{Conjecture I and Wall's Obstrugtion.}

Lemma 6. Suppose $X \subset R^{n}$ is a compact connected set which is a retract of some open subset of $R^{n}$, and $\varepsilon>0$. Then $\exists$ a finite $(n+1)$-complex $K . X \subset K \subset R^{2 n+3}$, such that $K^{n} \varepsilon$-deforms to $X$ in $K^{n+1}=K$, every element of $H_{n+1}(K, X) \approx H_{n+1}(K)$ is spherical, and $H_{n+1}(K)$ is generated by $\left\{f_{*}\left[H_{n+1}\left(S^{n+1}\right)\right]: f: S^{n+1} \rightarrow K\right.$ satisfies $\left.d\left[f\left(S^{n+1}\right)\right]<\varepsilon\right\}$.

This is proved by brute force construction and induction. Suppose for example, that $K$ is given as above, and it is desired to construct $L=L^{n+2}$ such that $L^{n+1}$ small-deforms to $X$ in $L . L$ is formed by attaching small $(n+2)$-cells to $K$. It must be shown that $\pi_{n+1}(K, X)$ is locally finitely generated and that $(n+2)$-cells may be attached in a manner that will allow $K$ to deform to $X$ by a slight movement (this is a difficult geometric construction).

THEOREM 3. Suppose $X \subset R^{n}$ is a compact connected set which is a retract of some open subset of $R^{n}$. Then Conj. $\mathrm{I}_{2 n+3}$ implies that $X$ has the homotopy type of a finite complex.

Let $K$ be as in Lemma $6, X \subset K \subset R^{2 n+3}, \widetilde{K}$ be the universal covering space of $K$, and $\pi=\pi_{1}(K)=\pi_{1}(X) . H_{n+1}(\widetilde{K})$ is a projective $\pi$-module and its element in the projective class group of $Z(\pi)$ is Wall's obstruction [9]. If $H_{n+1}(\tilde{K})$ is a free $\pi$ module, the free generators will allow $(n+2)$-cells to be attached to $K$ to determine $L$ such that $X \subset L$ is a homotopy equivalence. In the covering space this corresponds to killing $H_{n+1}(\tilde{K})$ without creating any $(n+2)$-cycles. Suppose Conj. $\mathrm{I}_{2 n+3}$ is true and let $W$ be a $(2 n+3)$-cell with $X \subset \operatorname{Int}(W)$. Then $K$ can be constructed to lie in $W$. We consider geometric groups associated with $W$, not with $K . G=G(W)$ will be generated by $\left\{p_{i}\right\}$ constructed as follows: Let $\left\{P_{i}\right\}$ be a collection of small cycles, $P_{i} \in Z_{n+1}(K)$, which generate $H_{n+1}(K)$, and let $p_{i} \in K \subset W$ be a point of the carrier of $P_{i}$. Let $H=H_{n+1}(K)$. Then $f: G \rightarrow H$ defined by $f\left(p_{i}\right)=$ the homology class of $P_{i}$ is a $\delta$-map. The conclusion of Conj. I gives a free basis for $H_{n+1}(K)$ and each basis element is carried by a small cycle. Since these cycles are small, they lift homeomorphically to the covering space where $\pi$ acts freely on them. 
Summary. Lemma 6 gives a $K$ where $H_{n+1}(K)$ is generated by small cycles. Conj. I is used to determine a free basis composed of small cycles. This free basis lifts to a free $\pi$-basis of $H_{n+1}(\tilde{K})$.

II. Noncompact Smale Theory.

Conjecture A. Suppose $M$ is a p.w.l. $n$-manifold $(n \geqq 6)$.without boundary topologically embedded as a closed subset of $R^{2 n+3}$. Then if $\varepsilon(x): R^{2 n+3} \rightarrow(0,1)$ is continuous, $\exists$ a continuous $\delta(x): R^{2 n+3} \rightarrow(0,1)$ such that if $C$ is a p.w.l. manifold topologically embedded as a closed subset of $R^{2 n+3}, \partial C=$ two components $M$ and $M_{1}$, and $C \delta$-deforms to $M$ and to $M_{1}$, then $\exists$ a p.w.l. homeomorphism $h: M \times I \rightarrow C$ with $h(x, 0)=x$ and $d[h(x, I)]<\varepsilon(x)$ for $x \in M$.

First consider the special case where $M$ is compact. This conjecture states that the $H$-cobordism is trivial whether $M$ is simply-connected or not; i.e., it states that $M \subset C$ is a simple homotopy equivalence. This is implied by Conj. $\mathrm{II}_{n}$ and Lemmas 3 and 4. Conj. A also says that $C$ is trivial in a controlled manner, i.e., $d[h(x, I)]<\varepsilon$. This requires reproving Smale theory using $\delta$-blocked matrices. The column operations necessary to diagonalize the matrix can be done locally and handles can be cancelled in a controlled manner. Thus Conj. $\mathrm{II}_{n}$ implies Conj. A for $M$ compact. For noncompact $M$, Conj. II must be generalized to consider infinitely generated geometric groups associated with infinite complexes. Since Conj. II is local in nature, this is only a slight generalization. Summary: A generalized Conj. II $_{n}$ implies Conj. A.

III. STABLE HomeOMOR Phisms.

CONJECTURE B. Suppose $f: R^{n} \rightarrow R^{n}(n \geqq 5)$ is a homeomorphism and $\exists$ an integer $k$ such that $f \times I: R^{n} \times R^{k} \rightarrow R^{n} \times R^{k}$ is stable [15]. Then $f$ is stable.

It suffices to consider the case $k=1$. A homeomorphism is stable iff it can be approximated by a p.w.l. homeomorphism [16]. Suppose $g: R^{n} \times R \rightarrow R^{n} \times R$ is a p.w.l. homeomorphism approximating $f \times I$. Move $g$ slightly so that $g\left(R^{n} \times 0\right)$ $\cap R^{n} \times 0=\varnothing$. Then $R^{n} \times 0$ and $g\left(R^{n} \times 0\right)$ bound an $H$-cobordism and using Conj. A, $f$ may be approximated by a p.w.l. homeomorphism. Thus Conj. A implies Conj. B.

IV. Diagonalizing Infinite Matrices.

THEOREM 4. If $M$ is a positive integer, $\exists$ a positive integer $N$ such that: If $a_{i, j}$ and $b_{i, j}$ are integers for $-\infty \leqq i, j \leqq \infty$, with $a_{i, j}=b_{i, j}=0$ for $|i-j|>M$ and $\left(a_{i, j}\right)\left(b_{i, j}\right)$ $=$ the identity matrix, then $\exists$ matrices $\left(c_{i, j}\right)$ and $\left(d_{i, j}\right)$ and an integer $k,-M \leqq k \leqq M$, such that

$$
\begin{aligned}
\left(c_{i, j}\right)\left(d_{i, j}\right)=\left(a_{i, j}\right), & \\
c_{i, j}=0 & \text { when } \exists n \text { with } 2 n N \leqq i \leqq 2(n+1) N \text { and } \\
& (j<2 n N+k \text { or } j>2(n+1) N+k), \\
d_{i, j}=0 & \text { when } \exists n \text { with }(2 n-1) N \leqq i \leqq 2(n+1) N \text { and } \\
& (j<2(n-1) N \text { or } j>(2 n+1) N) .
\end{aligned}
$$


This is analogous to Conj. $\mathrm{II}_{1}$ for the noncompact complex $K=$ Reals. The proofs of Conj. $\mathrm{II}_{1}$ and Theorem 3 are similar, except Theorem 3 is easier. Here is the idea for constructing $\left(c_{i, j}\right)$. Divide $\left(a_{i, j}\right)$ into finite blocks $\ldots A_{-2}, A_{-1}, A_{0}$, $A_{1}, \ldots$, throw away $A_{i}$ for $i$ odd and replace it by $B_{i}$ where $B_{i}$ is an identity matrix slightly smaller than $A_{i}$. Now the columns of $A_{2 i}$ are part of a basis for an infinitely generated free abelian group and, of course, so are the columns of $B_{2 i+1}$. The approach is to take their union and extend it to a complete basis-this corresponds to filling in the gaps between $A_{2 i}$ and $B_{2 i+1}$. Use the fact that the inverse matrix is bounded about the diagonal to show this can be done. This gives $\left(c_{i, j}\right)$, and $\left(d_{i, j}\right)$ is defined by $\left(d_{i, j}\right)=\left(c_{i, j}\right)^{-1}\left(a_{i, j}\right)$. The blocks $A_{2 i}$ in $\left(c_{i, j}\right)$ will cause $\left(d_{i, j}\right)$ to have blocks of the identity and thus automatically be broken into finite nonsingular blocks.

\section{BIBLIOGRAPHY}

1. J. H. C. Whitehead, Simplicial spaces, nuclei, and m-groups, Proc. London Math. Soc. 45 (1939), 243-327.

2. - Simple homotopy types, Amer. J. Math. 72 (1950), 1-57.

3. - Simple homotopy types, Lectures at Math. Institute, Oxford, 1955.

4. — Combinatorial homotopy. I, II, Bull. Amer. Math. Soc. 55 (1949), 213-245; 453-496.

5. J. Milnor, On spaces having the homotopy type of a CW-complex, Trans. Amer. Math. Soc. 90 (1959), 272-280.

6. - Whitehead torsion, mimeographed notes, Princeton Univ., Princeton, N. J., 1964.

7. - Two complexes which are homeomorphic but combinatorially distinct, Ann. of Math. 74 (1961), 575-590.

8. C. T. C. Wall, An obstruction to finiteness of CW-complexes, Bull. Amer. Math. Soc. 70 (1964), 269-270.

9. - Finiteness conditions for CW-complexes, Ann. of Math. 81 (1965), 56-69.

10. S. Smale, On the structure of manifolds, Amer. J. Math. 84 (1962), 387-399.

11. - Generalized Poincaré conjecture in dimensions greater than 4, Ann. of Math. 74 (1961), 391-406.

12. B. Mazur, Relative neighborhoods and the theorems of Smale, Ann. of Math. 77 (1963), 232-249.

13. E. Moise, Affine structures in 3-manifolds. IV. Piecewise linear approximations of homeomorphisms, Ann. of Math. 55 (1952), 215-222.

14. R. H. Bing, An alternate proof that 3-manifolds can be triangulated, Ann. of Math. 69 (1959), 37-65.

15. M. Brown and H. Gluck, Stable structures on manifolds: I-III, Homeomorphisms of $S^{n}$, Ann. of Math. 79 (1964), 1-58.

16. E. Connell, Approximating stable homeomorphisms by piecewise linear ones, Ann. of Math. 78 (1963), 326-338.

17. J. Stallings, "On infinite processes leading to differentiability in the complement of a point," in Differential and combinatorial topology, Princeton Univ. Press, Princeton, N. J., 1965, pp. 245-254.

18. T. Homma, On the embedding of polyhedra in manifolds, Yokohama Math. J. 10 (1962), 5-10. 
19. H. Gluck, Embeddings in the trivial range, Ann. of Math. 81 (1965), 195-210.

20. F. T. Farrell and W. C. Hsiang, $H$-cobordant manifolds are not necessarily homeomorphic, Bull. Amer. Math. Soc. 73 (1967), 741-744.

21. F. T. Farrell, The obstruction to fibering a manifold over a circle, Bull. Amer. Math. Soc. 73 (1967), 737-740.

\section{RICE UNIVERSITY,}

Houston, Texas 